\title{
Analysis of Dynamic Systems With Various Friction Laws
}

J. Awrejcewicz

e-mail: awrejcew@p.lodz.pl

e-mail: olejnikp@p.lodz.pl

Department of Automatics and Biomechanics, Technical University of Lodz,

Lodz, Poland
This survey is devoted to the significant role of various dry friction laws in engineering sciences. Both advantages and disadvantages of a frictional process are illustrated and discussed, but excluding the nature of friction. It is shown how the classical friction laws and modern friction theories exist in today's pure and applied sciences. Static and dynamic friction models are described. An important role of purely theoretical and experimental investigations in developing the appropriate friction models is outlined, placing an emphasis on new approaches (models proposed by Bay-Wanheim, Dahl, BlimanSorine, Lund-Grenoble, as well as atomic scale and fractal models, among others). Friction treated as a complex process being in interaction with wear, heat emission, and deformation is also discussed. Then the impact of dry friction models on current dynamical systems theory is reviewed. Finally, an application of friction to model a brake mechanism as a mechanical system with two degrees-of-freedom, including experimental and numerical analyses, is given. This review paper contains 254 references.

[DOI: $10.1115 / 1.2048687]$

\section{Introduction}

The relative motion of solid bodies with regard to adhesion is a disequilibrial process in which the kinetic energy is transformed into the energy of irregular microscopic movement. This phenomenon is related to energy dissipation (as heat, for instance) and leads to friction and wear in the contacting bodies area. Widespread occurrence of friction in technology and in everyday life is the reason for extensive scientific research that would result in widened theoretical knowledge of the friction process. The aim of such research is to provide a detailed comprehensive description of this phenomenon.

In contrast to many advantageous uses of friction, for instance, in metalworking, movement of vehicles, drive transmission with the use of frictional elements, even walking or vibration of strings in musical instruments, there are numerous negative aspects of friction in the form of noise, wear, and unpredictable behavior of various mechanisms. Special attention needs to be paid to harmful self-excited vibrations in engineering systems with friction that are associated with a periodic supply of energy from a constantly operating source that is controlled by the system's motion by means of a feedback mechanism. Such a relation leads to reciprocal reaction between the regulating device and the nonlinear vibrating system; thus, the self-excited system may control its own energy balance. As a result, despite inevitable losses, some nondisappearing periodic vibrations can be exhibited by the system.

Self-excited vibrations in kinetic junctions are usually harmful and undesirable, especially when in extreme cases they may result in destruction of a vibrating object. Among the phenomena in question are the vibrations caused by rotation of air around oscillating flexible connectors, strings, or coatings (vibrations of electric wires or a plane's wing's "flutter") [1-3]. Mechanical systems with coupled movements may generate self-excited vibrations at the expense of energy from the drive system. Such characteristics may be attributed to the "shimmy" vibrations, which cause snaking of a car's front wheels. Among typical examples of the occurrence of self-excited vibrations in nature are sounds of speech and the whistle of slender twigs in the wind. However, the same vibrations that cause such acoustic phenomena as a creak of old hinges or a loosened joint are associated with the negative characteristics of dry friction.

The disturbance in the fluency of motion caused by self-excited

Transmitted by Assoc. Editor J. Engelbrecht. vibrations resulting from an abrupt change of real sliding speed of frictional bodies is called a stick-slip effect. It can often be observed in measuring devices, precision tools, and during machining processes.

The basic property of friction conducive to stick-slip vibrations is the fact that the maximum friction force at the adhesion loss is larger than the force that occurs in the system during the slip phase at low relative speeds of sliding bodies. Mechanical devices with kinetic junctions characterized by friction of this type are exposed to additional mechanical vibrations, which in many cases cause disturbances in operation or damages. The occurrence of friction-excited vibrations in such systems as a knife and a support or a stock and a metal working machine results in worsening of the processed surface roughness.

Scientific treatises concerned with research on self-excited vibrations, including stick-slip vibrations, aim at thorough exploration of the essence of this phenomenon. An occurrence of selfexcited vibrations results from the lack of stability of the states of equilibrium or stabilized motion. Presumable presence of such vibrations in a kinetic slide junction should be detected because the machine, the states of equilibrium, may fall into undesirable vibrations leading to malfunction.

Therefore, various scientific research works that determine the characteristics of friction, study stability, or identify the parameters of friction with the dominating role of self-excited vibrations significantly contribute to general understanding and eliminating that phenomenon together with its usually unwelcome side effects on operating mechanical devices.

\section{Modeling of Dry Friction}

All mechanical and mechatronical systems that effectively use friction require appropriate design, which involves accurate understanding and detailed phenomenological description of the contact area. Excessive friction of the elements during vibration often causes decreasing precision of a mechanism's work and generates limit cycles. Therefore, various compensation techniques have been developed in order to improve dynamics and to minimize the negative influence of friction. The procedures employed in such cases usually consist of an accurate determination of conditions in a friction point, application of friction law, and determination of all movement coefficients. A correct theoretical description supported by an experimental model embracing all of the elements operating together lay basis for compensation of friction effects in vibrating systems. 
Unfortunately, a long-term research on external friction of solid bodies, which is common in every-day life and in technology as well, has not developed a general theory that would have fully explained this phenomenon. Some hypotheses, formulated at the turn of century, were based on the process of dry friction that belongs to the general theory of dynamic friction of solid bodies. The modern description of friction presented in Sec. 2.2 is based on well-known models. However, there are also models that approach the problem in entirely new ways.

Making a simplification all models in Sec. 2.2 treat static and dynamic friction as two separate processes from the dynamic modeling point of view. It is worth noteing that, in general, between static and dynamic friction there exists a region during which the solid rubbing bodies get a very small relative shift due to surface layers elastic deformations before the friction surfaces slip. The phenomenon may have great influence on the stick-slip behavior on certain conditions, especially when the slip phase is small enough.

2.1 Classical Friction Laws. It is a generally known fact that external dry friction occurs on nonlubricated surfaces of solid bodies that move relative to each other during adhesion. Research on friction conducted in the period between the fifteenth and the nineteenth centuries allowed Moore to formulate the "classical friction laws" presented below [4].

It was Leonardo da Vinci who concluded that the friction coefficient is not dependent on the nominal contact surface. He developed his theory from an observation of friction of a coiled rope stretched out by applying a force. Leonardo da Vinci's mechanical theory was based on the observed phenomenon of "the bodies varied capability to slip, which resulted in varied values of friction." The law formulated by da Vinci is limited in certain aspects because it is true only for the materials of determined yield points.

At the end of the seventeenth century Amontons formulated an observation-based dry friction law that claimed that the friction force was proportional to the normal force perpendicular to the contact surface of frictional bodies $(F=\mu N)$ with a dimensionless coefficient of proportionality $\mu$, independent of a load. In fact, the friction coefficient depends on the load as well as on the mechanical, geometrical, and chemical properties of the frictional surfaces. Despite the fact that Amontons' formula lacks accuracy, it has been widely applied in calculations today.

The next classical friction law derived from an observation that the static friction coefficient is larger than the dynamic friction coefficient, as a matter of fact, cannot be applied to elastic and viscoelastic materials either. That leaves us with one more classical friction law that claims that the friction coefficient is independent of the sliding speed. It is obviously a false statement since it makes the law applicable only to metals at low and medium relative velocities.

Mechanical theories of friction laid the foundation for further study of this complex phenomenon. Amontons' theory referring to the classical dry friction law was expanded by Coulomb in the eighteenth century by a dry friction law in the form of $F=\mu N$ $+C$ with correction parameter $C$ that allowed for the dependence of the friction force on molecular reaction of the frictional surfaces [5]. Coulomb assumed that for flat surfaces, correction $C$ has a constant value independent of the normal loading and the contact surface.

Mechanical theories of dry friction include also the theory of Bowden and Tabor [6]. It is based on an assumption that allows for plastic deformations of frictional solid bodies' real contact surface. The basic feature of this theory is the view on creation and destruction of junctions appearing on the contact surface, and the frictional resistance is defined as the sum of the resistances of cutting the joined asperities and the resistance of pushing the deformed material.

The knowledge on the phenomenon of dry friction was devel- oped due to molecular theories of Tomilson and Deryagin, mechanical-molecular theories of Kragelskiy, and energy theories of Kuznecov and Kosteckiy [7-9].

Epifanov [10] developed Bowden and Tabor's theory, however, his own friction theory was based on an assumption that a moving slide representing a single asperity of the surface cuts the buildup that forms in front of it.

2.2 Modern Description of Dry Friction. The process of external dry friction that occurs in various junctions of machines and mechanisms has been investigated with the utmost attention these days. The attempts to come up with a qualitative explanation of it by means of an appropriate mathematical notation face numerous difficulties related to the contact surface's complex structure, heat emission, and wear processes [11]. These problems do not close the list of all processes that occur in the adhesion area. Therefore, an analytical and experimental explanation of the character and the mechanisms of their occurrence is a priority in the investigation of discontinuous dynamic systems. That is why, in order to develop a friction model, mathematical equations that describe the frictional contact are formulated and experiments on true real systems with friction are conducted. The methods of mathematical analyses, numerical simulations, or empirical experiments are varied. The classical friction laws are applied when motion is only slightly affected by friction. Empirical experiments are conducted to verify the results of measurements with the use of analytical models. For the investigated frictional pairs and the conditions of motion, their own friction models (which take into account the specific conditions of the investigated junction) are formed. There are also numerous innovatory ways to apply the finite element method or fractal geometry $[12,13]$.

Because of the researchers' various approaches to the problem and their different methods of description, in what follows only the most important models of static and dynamic friction have been distinguished and described. The static friction models describe the dependence of the friction force on the relative sliding velocity, whereas the dynamic friction models take the form of differential equations, which also describe the friction in the stick phase of the frictional elements, i.e., when the measured relative sliding velocity is equal to zero.

\subsubsection{Static Models}

2.2.1.1 Numerical simulation. Vibrations excited by friction often cause problems in industrial mechanical devices, such as junctions of turbine blades, junctions of robots' parts, electrical engines' drives, water-lubricated bearings used in ships and submarines, machine tools, and brake and clutch systems. Vibrations of this kind are undesirable, and their side effects often affect the mechanical system's efficient operation in a negative way $[1,14-16]$.

The analysis of a system with one degree of freedom with friction in work [17] is based on the assumption of a harmonic exciting force and a standard excitation in the form of a belt moving at constant velocity. The main purpose of the work has been to determine in what way the system's response depends on the belt's velocity and the friction model. the numerical investigation has been based on the friction coefficient's discontinuous dependence on the relative sliding velocity $v_{r}$ (see Fig. 1) described by (i) Coulomb model and (ii) a model with the negative slope of friction characteristic in which the static friction coefficient $\mu_{0}$ decreases exponentially to the value of the dynamic friction coefficient $\mu_{k}$. In Fig. $1(b)$, the area marked by horizontal lines denotes low relative sliding velocities at which sticking of the mass occurs.

The friction characteristic described with Coulomb's law serve to show that the motion of the mass is stable during pure slips as well as during the subsequent stick and slip phases. The conditions in which it is possible to avoid the noise and stickslip vibrations in the system have been presented. The influence of the 

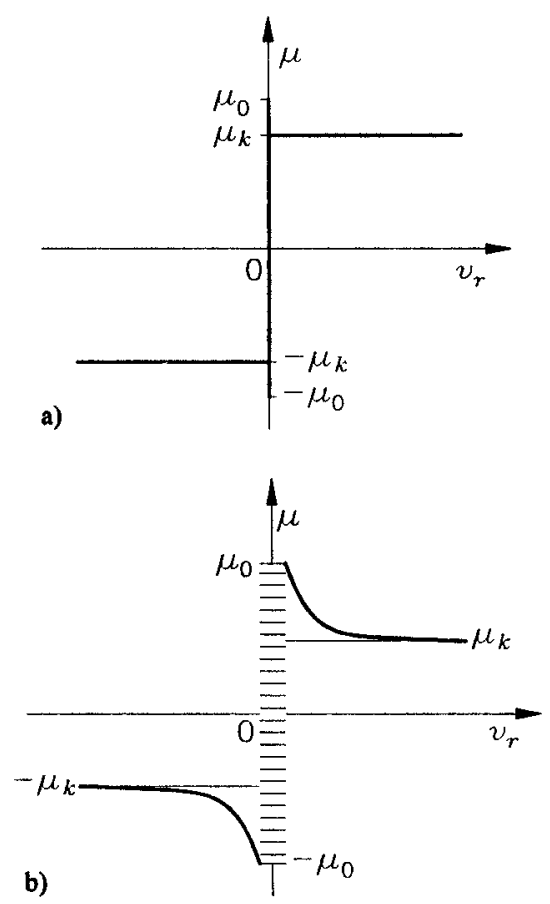

Fig. 1 Friction characteristics: (a) Coulomb law and (b) the exponential law

belt's velocity on the amplitude of transfer and the adhesion time has been investigated, and the results prove that beyond a certain value of the belt's velocity the oscillator's response does not change and the stick-slip vibrations vanish.

The transitory motion has also been analyzed to determine the conditions that should be satisfied by the system's initial state in order to obtain a sequence of pure slips and avoid stick states at the same time. In the case of the friction characteristics presented in Fig. 1(b), the relative velocity at which one can observe only the pure slip phases increases along with the fall of the exponential branches.

The work [17] also emphasizes a significant dependence of the system's response on the belt's velocity. It shows the period's doubling as well as $n$-periodical solutions and chaotic movements during the investigated system's motion. An additional analysis of a one degree-of-freedom system with harmonic excitation shows that the period's length and the number of "sticks" per motion's cycle may significantly increase.

The problem of stick-slip vibrations that occur during friction described with the Burridge-Knopoff earthquake model is presented in the works [18-22]. If the system presented in Fig. 2 includes more masses (the blocks connected by springs in series that vibrate on the belt) then modeling of stone blocks' slips during earthquakes will be possible on the grounds of the theory of seismic vibrations.

A numerical analysis of the Burridge-Knopoff model with the number of blocks reduced to two has been conducted considering the dependence on the dynamic friction force described by the following formula:

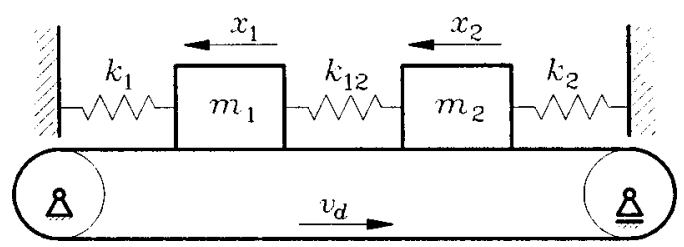

Fig. 2 Approximate Burridge-Knopoff model

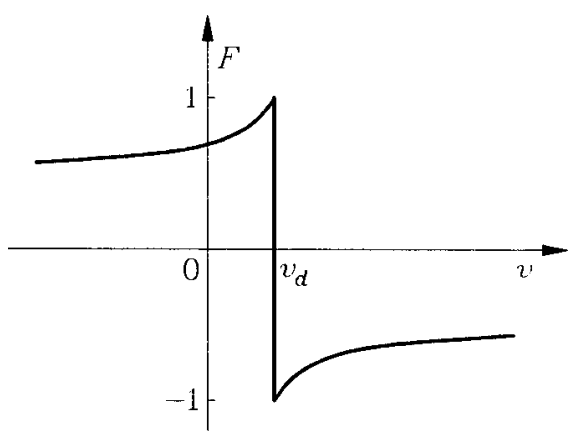

Fig. 3 Friction characteristics for the Burridge-Knopoff model

$$
F_{k}\left(v-v_{d}\right)=\left\{\begin{array}{cc}
\frac{1}{1-\gamma\left(v-v_{d}\right)} & v_{d}>v \\
-\frac{1}{1+\gamma\left(v-v_{d}\right)} & v_{d}<v
\end{array}\right.
$$

The dynamic friction force is usually a function of the relative velocity $\left(v-v_{d}\right)$ between the mass and the belt. The coefficient $\gamma=(0.4-3.4)$ has a positive value because the dynamic friction force (as it is presented in Fig. 3) decreases at small values of the relative velocity.

Introduction of the friction model described by the dependence that takes the form of formula (1) enables observations of nonstandard bifurcations and proves the existence of the attractive sets of solutions. An analysis of the obtained results leads to a conclusion that such assumptions as the lack of back slip and the existence of symmetry in the system may cause negative consequences in the form of inaccurate solutions. Considering all the conclusions drawn from the observation, some new assumptions have been made for a geophysical interpretation of the BurridgeKnopoff model.

Braking systems with anti-lock brake mechanisms $(A B S)$ prevent the wheels from blocking during a sudden application of the brake, which improves the directional stability and shortens the braking time. A two-dimensional nonlinear dynamic system with switching control is one of the simplest mechanisms with antilock brake systems. The nonlinearity in the system is manifested in the relation between the slide and the dynamic friction coefficient. An analysis of the dynamics of an anti-lock brake system with various methods of control has resulted in obtaining limit cycles of various shapes.

A physical model of an anti-lock brake system for a wheel, which includes nonlinear coefficients, is presented in [23] (see also the theoretical foundations presented in [24-27]). A very popular mathematical model of an anti-lock brake system is a system of three differential equations of the first order in the following form:

$$
\begin{gathered}
m \dot{v}=-F \\
J \dot{\Omega}=-\left(T_{b}-r F\right) \\
\dot{T}_{b}=u
\end{gathered}
$$

where $m$ and $v$ denote the vehicle's mass and velocity, $F=\mu N$ is the dynamic friction force (described by means of Coulomb law), which occurs between the wheel and the road, $N$ is the normal force, $J$ denotes the wheel's moment of inertia, $\Omega$ is the angular velocity of the wheel with radius $r$ (the distance between the wheel's axle and the road's surface), $T_{b}$ is the brake's torque on the wheel, and $u$ denotes the speed of the brake's torque's change.

The conducted experiments confirmed the existence of a non- 


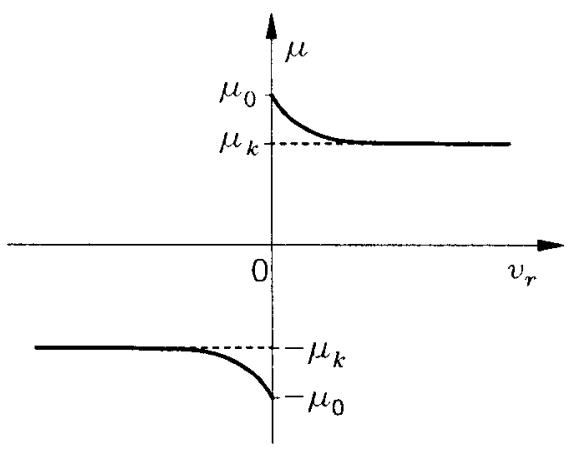

Fig. 4 Exponential law of friction versus relative velocity

linear relation between the dynamic friction coefficient and the wheel's slip $S$. The wheel's torque may be presented as

$$
\begin{gathered}
T_{e}(S)=r \mu_{k} N\left[1+\frac{(1-S) J}{r^{2} m}\right] \\
S=1-\frac{r \Omega}{v}
\end{gathered}
$$

The control in an anti-lock brake system consists in keeping the dynamic friction coefficient $\mu_{k}$ in the proximity of the boundary value $\mu_{c}$ causes such braking that creates the greatest possible friction force $F$ without blocking the wheel and slipping of the tire on the road's surface. The authors of the work also present an analytically numerical approach to obtaining periodical solutions of a piecewise nonlinear autonomous anti-lock brake system with boundary control.

Measurement of the static and dynamic friction coefficients is a frequent and well-known problem that appears during the investigations of systems with friction. Reference [28] contains a description of a tribometer (see also, [29]), which serves for the determination of the dependence of the coefficient of friction on a relative velocity between sliding bodies. It also enables to determine the static and dynamic friction coefficients for majority of pairs of frictional materials.

The Coulomb law has been applied during the simulation, and the friction coefficient dependent on the relative velocity has been approximated by a function in the following form (see Fig. 4):

$$
\mu\left(v_{r}\right)=\left(\left(\mu_{0}-\mu_{k}\right) \exp \frac{-\delta\left|v_{r}\right|}{\mu_{0}-\mu_{k}}+\mu_{k}\right) \operatorname{sgn}\left(v_{r}\right),
$$

where $\mu_{k}$ denotes the dynamic friction coefficient when the motion's relative velocity $v_{r}$ goes to infinity, $\mu_{0}$ is the static friction coefficient, and $\delta$ denotes a constant.

The results obtained from the measurements conducted in a true system and in a computer simulation have helped to prove that an appropriately adapted model of a tribometer and friction law depicted in Fig. 4 may serve as a perfect tool (as long as repeatability of results is obtained) for detecting and experimental visualization of friction.

The properties of a passive vibrations absorber with dry friction are significantly different from those of a classical linear absorber. An interesting phenomenon that can be observed in passive absorbers is their capability to dampen all forms of excited vibrations. It is affected by a small area of the friction characteristics and a well-evaluated static friction threshold. Reference [30] includes theoretical considerations and the results of experiments on the shape of the characteristics and its influence on the operation of a passive absorber.

A passive vibrations absorber is a mass-elastic system connected with the housing in order to control its vibrations forced by a periodical excitation. Figure 5 illustrates the positions of the

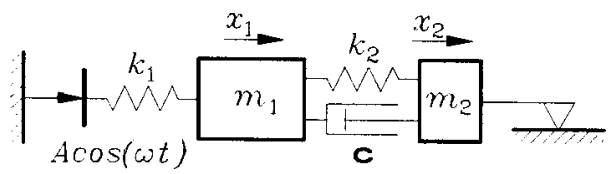

Fig. 5 Mechanical model of a passive vibrations absorber

masses and the connections of the absorber system.

Mass $m_{1}$ and spring $k_{1}$ are used to model the housing, while the absorber system is made of mass $m_{2}$, an elastic connector characterized by rigidity $k_{2}$ and viscous damping $c$. Mass $m_{1}$ is forced by a harmonic motion with amplitude $A$ and circular frequency $\omega$. Dry friction that occurs between mass $m_{2}$ and the guiding surface brings the problem down to an analysis of a strongly nonlinear mechanical system.

Classic study of the motion in mechanical systems with dry friction is, in most works, based on deterministic laws that have been defined as the product of the dynamic friction coefficient dependent on the relative velocity at the contact area and the normal loading dependent on time [1,15]. The authors of these works have made an assumption that the normal force is constant during motion and, by means of the characteristics described in Fig. 6(a)-6(c), they have also introduced a boundary value of the static friction force.

The simplest way to describe the friction force is the Coulomb

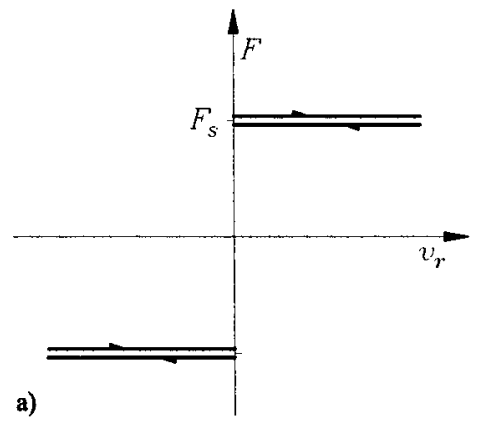

b)
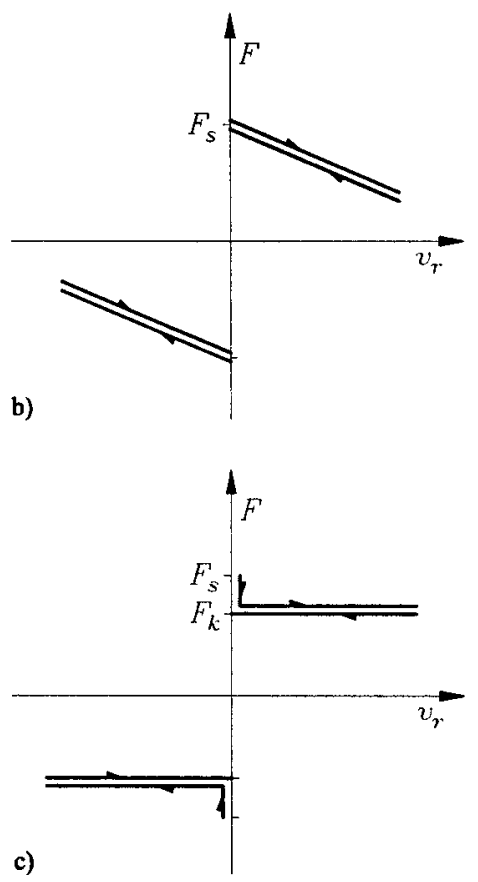

Fig. 6 Friction models: (a) Coulomb, (b) of a falling characteristics and (c) for $F_{s}>F_{k}$ 
law (Fig. 6(a)) according to which static friction force $F_{s}$ is equal to dynamic friction force $F_{k}$ dependent only on the motion's direction. In the case of the falling characteristics presented in Fig. 6(b) $F_{s}$ is still equal to $F_{k}$, but there is a linear dependence of the dynamic friction force described by a decreasing function on the velocity of mass $m_{2}$. The case presented in Fig. 6(c) involves a constant dynamic friction force that is smaller than the static friction force $F_{s}>F_{k}$.

An extremely interesting consideration has been conducted to see whether various models of friction induce any significantly different phenomena or responses of the passive vibration absorber. The most optimal use of the vibration absorber has been estimated on basis of the frequency characteristics. Comparisons of the shapes of the frequency response curves for a Coulomb model with the falling curve show little qualitative changes.

A change of the dynamic friction coefficient at the same assumptions as previously mentioned for the model of friction in Fig. 6(c) moves the damping of the high-vibration amplitude movements toward larger values of the static friction coefficient. If there is a small difference between these coefficients, then the system behaves in such a way as if Coulomb law described the friction (Fig. 6(a)). Practical application of the models of dry friction described above and a comparison of the obtained results prove that the system's response is affected by a small area of the friction characteristics and that the static friction coefficient influences the character of the transition between the stick and the slip phases.

Another study has also been conducted in order to observe the stick phase and to identify a dry friction model. The friction model has been identified (the shapes of its characteristics have been obtained as a result) not only by the change of such mechanical parameters as the relative velocity or the normal force, but also by the change of the frictional junction's material properties. In order to eliminate the additional influence of temperature and wear on friction, a special kind of brake pad friction material widely applied in engineering has been used [31].

The conducted experiments led to a conclusion that the efficiency of a passive vibrations absorber with dry friction is conditioned by the existence of the stick phases. The displacement during the slip phase should be big enough to make the mechanical energy dissipate from the system. However, these requirements are satisfied for large boundary values of the static friction coefficient (the static friction force is applied from the outside). Moreover, the analyzed shapes of the dynamic friction characteristics in the slip area slightly influence the dampening that occurs in the passive absorber.

In engineering dry friction often causes undesirable effects. It may cause some self-excited vibrations that generate noise and affect the durability of a system's elements. There are complex systems in which stick-slip vibrations may be observed [1]. The most interesting among them are the structures of some drilling elements on the deep-sea drilling platforms $[2,3]$.

The authors of [21] take up an analysis of the tensional stickslip vibrations that occur in rotational elements of the drills used on the deep-sea drilling platforms. Self-excited stick-slip vibrations caused by dry friction occur between a drill and the thinwalled pipes that lead the drill down into the seabed. They may cause damage to the working and fixing parts; that is why control systems are necessary to secure the entire construction against such vibrations.

In order to describe the friction that occurs in this system, the following formula of the friction characteristics is applied

$$
\mu=\frac{\mu_{0}}{1+\delta\left|v_{r}\right|}
$$

where $\delta$ denotes the parameter of the speed of the friction coefficient decrease along with the increase of the relative velocity $v_{r}$.

The use of the dependence described by formula (5) makes it possible to find such sets of parameters' values for which the stability of the points of equilibrium changes according to superand subcritical Hopf bifurcation. The occurrence of such a bifurcation of solutions influences formation of respectively stable and unstable periodical solutions.

In many mechanical systems the frictional elements tend to stick and slip occasionally, which often results in undesirable malfunctioning and uncontrolled behavior. Computer simulations of mechanical systems with dry friction are difficult to run due to strongly nonlinear friction characteristics in proximity of zero sliding velocity.

Tariku and Rogers [32] present two improved friction models. One of them is based on the method of equilibrium of forces. The other uses the elastic and dampening properties of such a system during the stick phase. The two models have been tested in many one- and two-dimensional systems with damping and elasticity and with excitation changeable in time and normal contact forces using the same methods of study.

Karnopp [33] has used the relation of the dynamic friction force during the slip formulated by Coulomb. However, the friction force during the stick phase has been determined on basis of the equilibrium of forces. A rigid body becomes physically stuck during friction if the relative velocity and the acceleration tangential to the friction surface equal zero. Since zero values of those variables cannot be obtained in numerical calculations, only small velocity "windows" are defined (for example, when the relative velocity is embraced within range $(-0.001-0.001))$, in which an assumption that the mass becomes stuck is made.

Some piecewise continuous analytical solutions have been compared to the solutions of other friction models based on equilibrium of forces and the elastic-dampening properties of the system during the stick phase. A method that enabled us to estimate the dimensions of a velocity "window" in friction Karnopp model has been developed $[33,34]$. The results obtained from the tests of the proposed method show that the new algorithm of the equilibrium of forces used for estimation of the velocity during the stick phase produces smaller errors in comparison to the original method. Moreover, the algorithm does not produce sharp "peaks" at the beginning of the stick phase.

The method developed by Karnopp [33,34] for the equation of motion:

$$
m \ddot{x}+d \dot{x}+k x=A \cos (\omega t)+F
$$

can be described in the following way:

1. If $|\dot{x}|>\dot{x}_{1}$, then a body with mass $m$ remains in the slip phase and the dynamic friction force is described by equation $F_{k}$ $=-\mu_{k} N \operatorname{sgn}(\dot{x})$.

2. If $|\dot{x}| \leqslant \dot{x}_{1}$, then the "net" friction force affecting the mass can be calculated by means of formula $F_{\text {net }}=A \cos [\omega(t$ $\left.\left.+t_{0}\right)\right]-(m \ddot{x}+d \dot{x}+k x)$

3. If $\left|F_{\text {net }}\right|>\mu_{0} N$, then a body slips and $F_{k}$ is determined in the way as in 1 .

4. If $\left|F_{\text {net }}\right| \leqslant \mu_{0} N$ then mass $m$ becomes stuck and is affected by force $F_{k}=-F_{\text {net }}$. The other constants $d, k, A$ and $\omega$ in (6) denote the damping coefficient, the coefficient of rigidity, the amplitude, and the frequency of the exciting force, respectively. Moreover, if the value of $\dot{x}$ is very small and $\ddot{x}$ $=0$, then regardless of the equilibrium of forces, the value of $F_{k}$ may be accurately approximated by means of: $F_{k} \approx$ $-A \cos \left[\omega\left(t+t_{0}\right)\right]+k x$.

The criterion of stick detection in the described method consists of the observation of the change of the sliding velocity's sign rather than in the attempts to define a velocity "window." An alternative criterion may be an external net force less than the maximum friction force. When the system becomes stuck, the sliding velocity falls to zero and changes its sign. Defining a lowvelocity window allows the algorithm to omit the stick phase. The use of the modified model results in lower constant stick velocities 
in comparison to Karnopp's method.

As soon as sticking is detected in the Antunes model [32], a rigid spring and a damper are fitted along the motion's direction. Thus, the friction force during sticking can be notated as a sum of the elasticity and damping forces. The method of stick phase detection consists of simultaneous fulfilling the following conditions: (i) the sliding velocity changes its sign, (ii) the elasticity and damping force during sticking is less or equal to the friction force during slipping. The stick phase is over as soon as the elasticity and damping force overpowers dynamic Coulomb friction force.

In the quoted publication [32], the original Antunes' model (which includes the elasticity and damping force) is modified according to the following criteria: (i) the dynamic and static friction coefficient are taken into consideration; (ii) because of a very small calculation time step, the original algorithm of Antunes model applied in the work sometimes detects sticks during the sliding velocity's change of direction even when the net friction force $F_{\text {net }}$ is greater than the maximum friction force. In order to avoid such a temporary sticking, the model has been modified by imposing a condition that the sticking may occur only when the net force is less than the maximum friction force.

The modified friction model with elasticity and damping forces presented by the authors of the discussed publication assumes simultaneous fulfilling of the following criteria in order to detect sticking: (i) the change of the velocity's sign, (ii) the external net force must be less than the maximum friction force, (iii) the additional elasticity and damping force related to friction during sticking should be less than the maximum friction force. When sticking occurs, the algorithm activates the elasticity and damping force, which makes the system return to the state a few time steps before and carries the calculations on until the friction force during slipping matches up with the friction force from the additional virtual spring and damper. The algorithm converges again in the point of zero velocity with the still active elasticity and virtual damping. It is necessary to add that the additional elasticity and damping tangent to the motion's direction applied during sticking are by no means related to the real physical values.

Numerous simulations confirm the fact that modified algorithms based on the equilibrium of forces and application of an additional elasticity and damping force significantly improve the existing methods. As a result, the stick-slip problem in dry friction systems can be solved with greater accuracy. During sticking, an equilibrium-of-forces model keeps constant low velocities, whereas a model with an additional elasticity and damping force gradually lowers the values of the stick velocity and thus more accurately approximates the zero velocity in the stick phase.

There are certain difficulties in estimation of a window at determining the sliding velocity's zero value in Karnopp model with equilibrium of forces [33,34]. Therefore, the stick phase is often omitted in the calculations and too high sliding velocities are reached at high velocities of the base (frame). The improved method is not based on determining the velocity windows, and it keeps low sliding velocities, which are usually much lower than in the previously mentioned Karnopp model, during the change of sign.

2.2.1.2 Experiment. A mechanical object consisting of a system of frictionally interacting masses that viscoelastically oscillate on a transmission belt is presented in $[35,36]$. The aim of the research was to formulate a model of dry friction that would describe the problem of stick-slip and quasi-harmonic vibrations. The research included the motion's relative velocity, acceleration, adhesion time, and velocity of the friction force increase at the moment preceding the stick-slip transition.

The empirical research aiming at determining the friction model's parameters was conducted at the experimental rig used to investigate the vibrations of self-excited systems. The rig consisted of the following elements: a mechanical system made of a system of masses connected by springs and an optical-electronic measuring system used for visualization, collecting, and digital

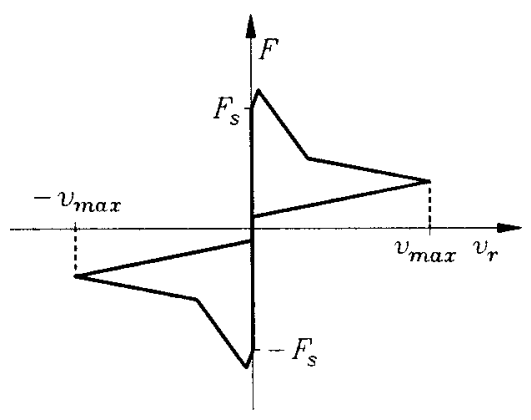

Fig. 7 General form of a dry friction model ( $F$-friction force, $v_{r}$-relative velocity, $F_{\mathrm{s}}$-friction force at the moment of breaking, $v_{\text {max }}$-maximum relative velocity)

processing of data.

The experiment brought results in the form of the masses' coordinates, which allowed determining the motion's dynamic characteristics. The obtained dependencies of relocation, velocity, and acceleration on time allowed determining the static and dynamic friction force in the function of the investigated system's parameters.

The investigation of the dynamic friction force in the function of the relative velocity and the acceleration's sign, as well as the investigation of the static friction force dependent on the sticking time and the velocity of the force change made it possible to formulate a dry friction model (Fig. 7) for a steel-polyester frictional pair.

The experimental research, modeling, and computer simulation of the vibrations of a self-excited system in the case of the assumed model of dry friction proved that the belt's velocity significantly influences the system's behavior. The analysis of the occurrence of stationary solutions proved that there are stick-slip relaxation vibrations in the system and that the system's vibrations are strongly influenced by the static characteristics of friction. Along with an increase of the belt's velocity $(>0.25)$ one can observe a transition between the qualitatively new vibrations types of various frequencies, which is characteristic for supercritical Hopf bifurcation. At further increase of the belt's velocity $(>0.4)$, the self-excited vibrations disappear and the system approaches a stable slip in which the relative velocity assumes a constant value equal to the belt's velocity. In spite of the occurrence of such ranges of the belt's velocities at which the static or the dynamic friction force is more important the system's instantaneous motion depends on both characteristics of friction due to the model's sensitivity to the system's history. This phenomenon is characteristic for friction models with memory.

An analysis of self-excited vibrations in mechanical systems with many degrees of freedom was conducted in [37,38]. The experiments showed that a steel plate modeling a superficial contact of frictional bodies that was connected by elastic elements with a rigid body with three degrees of freedom vibrated almost harmonically with such velocity amplitude that a harmonic linearization of the sgn function occurred. It was assumed that the mass contacts of the frictional bodies in the feed motion systems of heavy machine tools might perform a similar role.

2.2.1.3 Mathematical approach. Considerations on the mathematical approach in the study of discontinuity, including the analysis of complex phenomena of friction, are presented in [39-45]. Assuming multivalent representations (for a sgn function, for instance) and using the equations of discontinuous oscillators as simple differential inclusions dependent on small parameters, the manifold of periodical solutions and sufficient conditions of their occurrence are investigated.

Another example of a work that presents numerical solutions and mathematical considerations on the structure of the bifurca- 


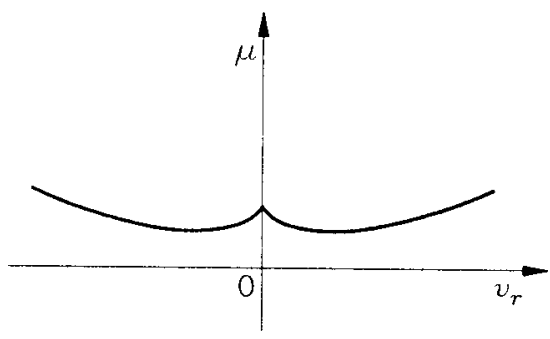

Fig. 8 Friction characteristic for periodically excited oscillator

tion parameters of nonsmooth oscillators with dry friction is Ref. [45]. The authors investigate a periodically excited oscillator with dry friction with one degree of freedom in which the exciting force is presented in the harmonic form of $A \cos (\omega t)(A$ and $\omega$ are the amplitude and the exciting force frequency, respectively). The elasticity proportional to relocation $x(t)$ is modeled in accordance to Hook's law; the dry friction force is described in accordance to Coulomb law; and the change of the relative velocity is described by function $\operatorname{sgn}(v(t)-1)$.

With the use of the second Newton law of dynamics, the following differential equation of the second order has been obtained:

$$
\ddot{x}+x+\mu_{k}[\mu(1)+\operatorname{sgn}(v-1) \mu(v-1)]=A \cos (\omega t)
$$

where $\mu(1)$ is the value of friction coefficient when the velocity is equal to 1. Dynamic friction coefficient (see Fig. 8) is described by the following relation:

$$
\mu_{k}=\frac{\mu_{0}-\mu_{1}}{1+\lambda_{0}\left|v_{r}\right|}+\mu_{1}+\lambda_{1}\left|v_{r}\right|^{2}
$$

where $\mu_{0}$ is the static friction coefficient and $\mu_{1}, \lambda_{0}, \lambda_{1}$ are constants.

The discussed work presents a difference between continuous and discontinuous dynamic systems that is related to the existence of Lapunov exponents. Interestingly enough, the assumed threedimensional space degenerates because of the trajectory's return to a discontinuous surface of a lower dimension. Therefore (as the calculations confirm), the first Lapunov exponent converges to infinity. The second exponent is equal to zero by assumption (the system evidently depends on time); therefore, the motion's character is entirely described by the third Lapunov exponent. Such a situation is beneficial because it allows using Eq. (7) to reduce the investigation of the system to the investigation of a onedimensional Poincaré map. More-over, based on the obtained bifurcations of solutions, a conclusion was drawn that there are still many structures of that type that require thorough investigation and explanation.

A generalized Bay-Wanheim friction model that describes the mixtures of thin lubrication films was applied in industrial programing utilizing the finite element method [46,47]. A threedimensional axial-symmetric problem was presented and subsequently verified during the tests of circular compression by comparison of the obtained empirical and analytical results. The Bay-Wanheim model was also used to describe friction that occurs during the rotary and slip motion of a processed sample with low and medium loading in the adhesion area. The methodology of friction coefficient identification was developed, and the advantages as well as the disadvantages of Bay-Wanheim model and its supremacy over the Coulomb model were pointed out.

The friction model proposed by Bay-Wanheim $[48,49]$ is characterized by lubrication processes that occur between the fixed base and the sample that rotates and slides on it. It is described by the following relation:

$$
\frac{P}{P_{\max }}=-\mu \alpha \frac{v_{r}}{\left\|v_{r}\right\|}
$$

where $P$ emphasizes the reduction of the pressure force that appears during friction with respect to the coefficient of the real contact area $\alpha$ and an average friction coefficient $\mu, P_{\max }$ is a maximum pressure force during pure shearing, and $v_{r}$ denotes the relative velocity. It is essential to add that the model utilizes the material's physically nonmeasurable roughness. Coefficient $\alpha$, defined as the ratio of the real and measured contact surfaces, increases along with the increase of the pressure adequate to the type of interactions on the rough surface, and it depends on the assumed theory of elasticity.

Identification of the average friction coefficient $\mu$ (in formula (9)) in the Bay-Wanheim model is conducted according to a reverse approach, which depends on comparison and verification of empirical results with the use of the finite element method. The method has also been applied to compare the Bay-Wanheim model to the Coulomb model of friction. A procedure based on an assumption that the pressure force at the contact area is independent of the friction coefficient is applied to determine the friction coefficient for Coulomb law. Moreover, it is assumed that for a friction coefficient smaller than 0.15 the deflections of the effective plastic deformation are sufficiently small and may become independent of this coefficient.

Use of the Bay-Wanheim model leads to a more accurate estimation of the average friction coefficient $\mu$, and, subsequently, to a better determination of the fields of plastic deformations in the contact area proximity. Combination of the sample's rotation and slide with the generalized friction Bay-Wanheim law accurately represents the conditions in the frictional surfaces' adhesion area.

Works $[12,13]$ deal with the problems of fractal geometry and fractal behavior during unilateral contacts. The adhesion surfaces and the friction laws that apply to contact surfaces are modeled by means of fractal geometry. A fractal nature of the applied friction laws includes randomly located asperities on the contact surface that influence the friction force.

The fractal law and the fractal contact surface in a fractal model were examined in the form of two different functions yielded as a result of a fractal interpolation. With regard to these assumptions, the fractal friction law was approximated with a sequence of nonmonotonic, usually multivalent, $C^{0}$-class curves. The effectiveness of the numerical analysis of all nonmonotonic problems was improved by the use of the advanced solution methods that approximate a nonmonotonic problem with a sequence of monotonic problems. The work describes some numerical applications of the advanced solution methods for a static analysis of structures with cracks. The analysis employed fractal geometry and fractal friction laws formulated for friction contact surfaces with cracks.

The authors were mainly focused on the laws' fractal nature and their relation with the friction contact surface's fractal geometry. The application of fractal geometry allowed one to define the contact surfaces with cracks and to assume the friction mechanism.

A variational formulation of the fractal friction problem was based on a sequence of semi-variational problems; however, the adhesion surface' fractal approximation was made first. Unilateral contact conditions were assumed in a normal direction to the adhesion surface, whereas the fractal friction law (the characteristic of which is presented in Fig. 9) was assumed tangentially to it.

The assumption of the adhesion surface's fractal geometry and its fractal behavior in the problem of the unilateral contact led to yielding a correct model. The model accurately described the complicated geometry of a cracked surface with instantaneous loading and relative relocation leaps caused by partial damage to the adhesion surface. The velocity of the algorithm's convergence to the solution of the friction problem in a system with a fractally modeled structure strongly depends on the fractal friction law and 


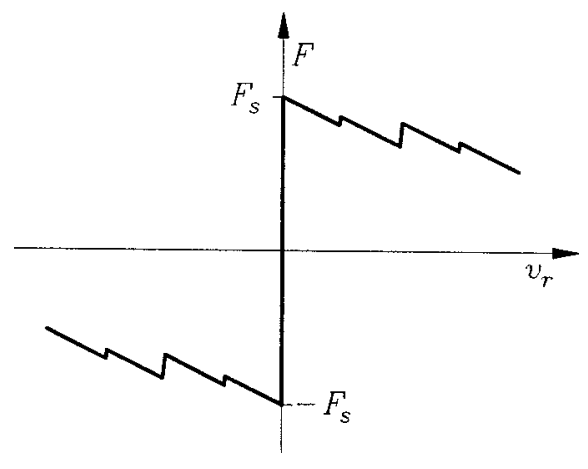

Fig. 9 Fractal friction model for an unilateral contact

the fractal dimension of the adhesion surface. The character of the generated loading additionally influences the velocity of convergence of the proposed algorithm.

2.2.1.4 Machine and mechanism junctions. The junctions in machines and mechanisms in which one can observe clearances and friction may be modeled as systems of nondeformable bodies connected by elastoplastic systems (the so-called multimass discrete systems) with unilateral constraints.

One of the most important characteristics of bolted joints is their capacity for damping as a result of friction between the parts of the joints. Extensive reviews of this subject by Gaul and Nitsche [50] have been completed. Their review article indicates approaches for describing the nonlinear transfer behavior of bolted joint connections. From a theoretical point of view, the carefully made overview of modeling issues [51-53] is very useful and numerically applicable. The analyzed cases include classical and practical engineering models. Constitutive and phenomenological friction models describing the nonlinear transfer behavior of joints are discussed [54,55]. Hertzian contact [56], the nonlinear relationships between friction and relative velocity in the friction interface, and a few solution techniques [56-59] commonly applied to friction-damped systems have been presented.

Works [60-62] present how a three-degrees-of-freedom mechanical system with friction and unilateral contacts was used to investigate the conditions of the transient states' occurrence. Discontinuous characteristics of friction and contact laws served to determine the transient states observed in machine and mechanism kinetic junctions.

The discussed work studied the following configurations of unilateral constraints: separation, stick contact, and slip contact. For a mechanical system with $n$ unilateral constraints, the number of all possible combinations of all constraints' states equals $3^{n}$. Thus, for the systems of a great number of unilateral constraints, the number of combinations suddenly rises. Only one of them fulfills all of the well-defined kinetic and kinematic conditions.

Formulation of the rigid body's dynamics equations and the contact law, and finding solution to the contact problem were accompanied by the linearization of the static friction cone (with the use of the Coulomb law). The characteristic of dry friction presented in Fig. 10 was applied to formulate the equations of the normally and tangentially directed constraints (using the notation of accelerations).

A preliminary theory (including an extended method of Lagrange multipliers) for the problem of a three-dimensional contact was given, and subsequently an oscillator with dry friction made of one rigid body (placed on a slant surface), on which three unilateral constraints were placed, was analyzed. The rigid body with mass $m_{1}$ placed on the slant surface was periodically excited by a rotating mass $m_{2}$ with unbalance $e$. The contact surface's inclination angle $\phi$ and the exciting mass' angular velocity $\Omega$ (Fig. 11) were assumed. An experiment gave evidence for occurrence of a stick-slip phenomenon during the motion. The oscilla-

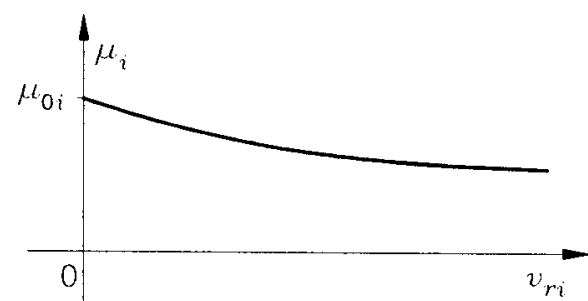

Fig. 10 A decaying characteristic of dry friction for $i$ th constraint

tor's locations were also measured and the results (obtained on basis of the previously applied theory for multimass systems with unilateral constraints) were compared with a computer simulation.

Investigation of the stick-slip phenomena in rigid multimass systems with unilateral friction contacts allows one to find an explanation of certain difficulties that occur in three-dimensional systems. They are especially visible when the behavior of one junction affects the other junctions. The analysis of such a case based on the dynamics of constraints leads to compatibility of the stated problem.

As it turns out, the use of a linear formulation of the problem (the linearization of the friction cone) does not result in finding only one combination of the states of all constraints, if the direction of the friction force that occurs in a constraint instantly after the transition from the stick phase to the slip phase is unknown. Such a situation leads to a nonlinear completion of the problem that may be solved by means of a few methods and algorithms roughly presented in this work. Additionally, if the conditions on the contacts are reciprocally dependent, then certain difficulties with a mathematical formulation of the stated problem appear.

The methods discussed in the quoted work, including Lagrange's extended method of multipliers, may be applied to multiple impacts with friction. Coulomb's impact model with friction is extended into a three-dimensional multiple impact with friction to be subsequently solved with the use-modified method of Lagrange multipliers.

2.2.1.5 Atomic scale. The friction force in the friction model presented below on the atomic scale [63] is constant for small values of the motion's velocity. A study conducted with the use of a microscope for various forms of carbon (diamond, graphite, and amorphous carbon) was focused on investigating the influence of velocity on the point contact with friction. The results show that the friction force is constant in a vast range of sliding velocities (nanometers to micrometers). The use of an FFM microscope-a more developed scanning force microscope (SFM) - allowed one to investigate the frictional properties of the point contact on the nanoscale. The behavior observed during the friction process was significantly different from the behavior characteristic for the macroscopic friction models. An especially interesting observation was that friction forces were proportional to the real contact surfaces, which, in turn, were not proportional to the forces exerted by the adhesion surface loading.

If the second derivative of potential with a negative sign after relocation is larger than constant elasticity (between the mass and

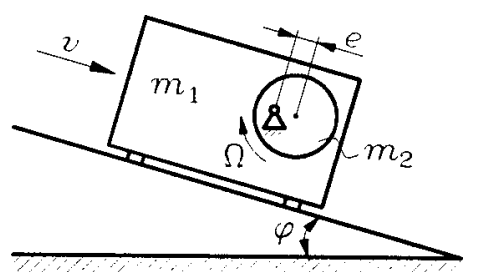

Fig. 11 Oscillator on an inclined plane 


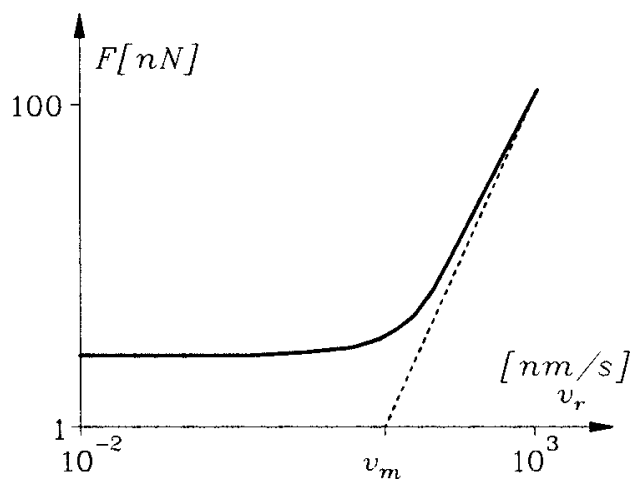

Fig. 12 Friction force $F$ as a function dependent on sliding velocity $v_{r}$

an atomic chain that is parallel to its motion and generates a potential's field), then some unexpectedly significant changes of relocation appear in the form of discontinuous stick-slip movements (leaps from one potential's minimum to another). At high velocities, the motion is dominated by viscous damping and the friction force at that time is proportional to the sliding velocity (see Fig. 12).

For low sliding velocities, friction force $F$ is determined by dissipation of energy and, thus, it is constant in the range of such velocities. The more the sliding velocity increases, the more the friction force is described by a linear dependence (the straight line corresponding to viscous damping that crosses point $v_{m}$ on the $v_{r}$-axis). Summing up, the work proves that macroscopic friction laws are not applicable on the microscopic scale.

2.2.2 Dynamics Models. Friction force in static models depends on sliding velocity. However, there is another approach according to which friction, as a phenomenon changeable in time, should be described with differential equations and viewed as a dynamic system. The description of friction with the use of differential equations is often applied to control oscillating systems with friction [64-66].

Dahl $[67,68]$ suggests a simple model of controlling systems with friction. The conclusions drawn from his experiments suggest that some microscopic irregularities located in the frictional surfaces' contact area are the reason for the occurrence of dry friction (according to the classic mechanics of solids). The starting point for Dahl's model is the curve representing the relation between the pressure force and the deformation $[69,70]$ presented in Fig. 13 .

Dahl suggests notating the curve in Fig. 13 as a differential equation of the following form $[71,72]$ :

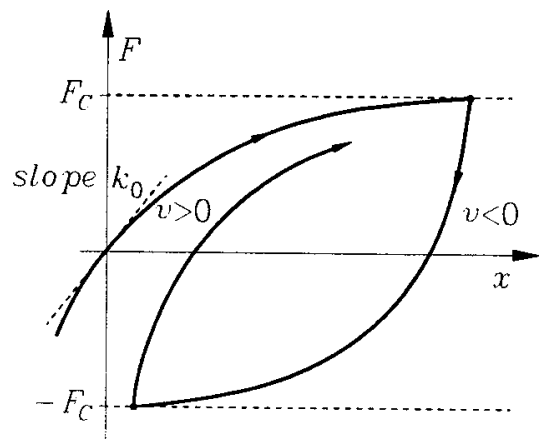

Fig. 13 Relation between pressure and deformation

$$
\frac{d F}{d x}=k\left(1-\frac{F}{F_{C}} \operatorname{sgn} v\right)^{\beta}
$$

where $x$ is the relocation, $v$ denotes the motion's velocity, $F$ describes the friction force, $F_{C}$ denotes friction Coulomb force, $k$ is the coefficient of rigidity, and $\beta$ (usually equal to one) denotes the shape parameter of the curve representing the relation between the pressure force and the deformation (stress-strain characteristics). Absolute value of the friction force will not be larger than $F_{C}$, if it satisfies condition $|F(0)|<F_{C}$.

It is essential to note that friction force in the Dahl model is only a function of relocation and the sign of velocity $[72,73]$. Such a model may be also described in the domain of time by the following transformation:

$$
\frac{d F}{d t}=\frac{d F}{d x} \frac{d x}{d t}=\frac{d F}{d x} v=k\left(1-\frac{F}{F_{C}} \operatorname{sgn} v\right)^{\beta} v
$$

If we assume that $\alpha=1$ and substitute $F=k z$, then we obtain the following system of equations:

$$
\begin{gathered}
\frac{d z}{d t}=v-\frac{k|v| z}{F_{C}} \\
F=k z
\end{gathered}
$$

A model described with (12) used to be widely applied in aviation industry, but presently it has been commonly used to describe friction in ball bearings. The system of Eqs. (12) efficiently "avoids" the numerical inconveniences that appear in static models. Often applied in compensation of the effects of friction, the system is not faultless, since it does not enable to describe a stick-slip motion or allow for Stribeck effect.

Several types of Bliman-Sorine models are presented in $[74,75]$. On the basis of the experiments conducted by Rabinowicz [76], they assume that friction depends on the sign of velocity $v$ and variable $s$ notated as

$$
s=\int_{0}^{1}|v(\tau)| d \tau
$$

The Bliman-Sorine model is represented by the following system of differential equations:

$$
\begin{gathered}
\frac{d x}{d s}=B x+C v \\
F=D x .
\end{gathered}
$$

The complexity of this model, as well as the previous one, depends on the dimension of the state's space. The description of friction with an equation of the first order is, in this case, identical to Dahl's approach-there is no stick phase in consequence. However, if the constants included in Eq. (14) assume the following form:

$$
B=\left(\begin{array}{cc}
\frac{-1}{\eta \varepsilon_{f}} & 0 \\
0 & \frac{-1}{\varepsilon_{f}}
\end{array}\right), \quad C=\left(\begin{array}{c}
\frac{f_{1}}{\eta \varepsilon_{f}} \\
\frac{-f_{2}}{\varepsilon_{f}}
\end{array}\right), \quad D=\left(\begin{array}{ll}
1 & 1
\end{array}\right)
$$

where $\eta, \varepsilon_{f}, f_{1}, f_{2}$ are certain parameters of the model, then a stick-slip motion can be observed and system (14) functions as a link between Dahl models for low and high relative velocities.

Bliman and Sorine [75] prove the dissipative character of the first- and second-order models and also show that if constant $\varepsilon_{f}$ is close to zero then the system of equations of the first order approximates classic friction Coulomb model, and the differential equation of the second order additionally allows for the stick- 


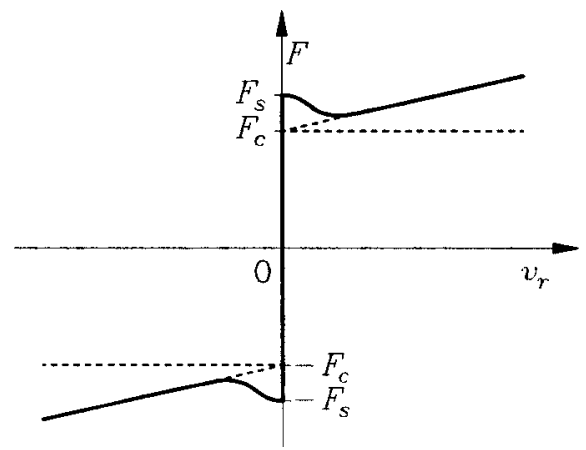

Fig. 14 Dynamic friction model

phase in it.

Work [77] determines the relation between the dynamic friction model presented in it and standard models of dynamic friction. The authors propose two classes of friction models to control and analyze mechanical systems: (i) a dynamic friction model, and (ii) Lund-Grenoble model.

Models of the first type include the dynamic friction models in which friction force depends on sliding velocity. As it was mentioned in Sec. 2.1 Coulomb [5] was the first to make observations of friction. The study of a loaded element that slid on a smooth surface at various velocities showed that viscous friction (in contrast to dynamic friction) slightly influences the phenomenon's character. Dynamic friction is almost constant and oppositely directed toward the motion's direction. The discussed work describes friction with the use of Coulomb law and denotes it as $F_{C}$ (Fig. 14).

The boundary static friction force that occurs during the sliding element's transition from the stick phase to the slip phase is called "a breaking force" and denoted as $F_{S}$ (sometimes $F_{S}=F_{C}$ ). The following relation describes the dynamic friction force in the analyzed equation of motion:

$$
F=k_{2} v+k_{0} g(v) \operatorname{sgn}(v)+(1-|\operatorname{sgn}(v)|) \operatorname{sat}\left(F_{u}-F_{g}, F_{s}\right)
$$

where

$$
\operatorname{sat}\left(F_{u}-F_{g}, F_{s}\right)= \begin{cases}F_{S}, & F_{u}-F_{g}>F_{S}, \\ F_{u}-F_{g}, & -F_{S} \leqslant F_{u}-F_{g} \leqslant F_{S} \\ -F_{S}, & F_{u}-F_{g}<-F_{S} .\end{cases}
$$

In the above $F_{g}$ is the gravity force, whereas $k_{0} g(v)$ includes Stribeck effect [28,78] (see also Sec. 2.2.1.1), and $k_{2} v$ describes the viscous friction that occurs at high sliding velocities.

A sgn function in the friction Coulomb model serves as a switch between three different models for negative, zero, and positive velocities. The basic assumption is that friction force $F$ depends on sliding velocity $v$ and force $F_{u}$ acting from the direction of the medium.

The Lund-Grenoble model governed by equation

$$
\begin{gathered}
F=k_{0} z+k_{1} \dot{z}+k_{2} v \\
\dot{z}=v-\frac{|v|}{g(v)} z
\end{gathered}
$$

assumes that frictional contact occurs between two porous flat surfaces. Variable $z$ denotes an additionally introduced state that models the average deflection of the porous surfaces. Porosity deflection of both surfaces generates friction force $k_{0} z+k_{1} \dot{z}$, where $k_{0}$ and $k_{1}$ are the coefficients of rigidity and damping, respectively.

The two friction models presented above differ from each other in their complexity. The dynamic friction model is relatively simple to use, but it turns out it cannot be differentiated during analyses and simulations. On the other hand, the Lund-Grenoble model is continuous and due to the possibility of using the Lipschitz condition, it enables one to find a solution for the known initial conditions. Its primary fault is that it introduces an additional immeasurable variable $z$ that ought to be taken under consideration during calculations.

A singular perturbation model described in the discussed work [77] binds the models mentioned above. Thus, they may be represented as a system of equations with a coefficient that functions as a transition from one model to another. The binding coefficient in the proposed notation of the singular perturbation model determines similarity between the Lund-Grenoble model and the dynamic friction model. According to the results of the analysis of cases $F_{C}=F_{S}$ and $F_{C} \neq F_{S}$, the differences described by the coefficient of perturbation between the two friction models are significant (on basis of the relation between the friction force and the sliding velocity obtained from a numerical simulation) only for low sliding velocities. If the angular velocity is high ( $v$ $>1.5 \mathrm{rd} / \mathrm{s}$ ) and constant, then the dynamic friction model appears to be more effective. However, when the sliding velocity is low, but changeable in time, then the two considered models describe friction with approximately the same effect.

Some interesting considerations that focus on the study of friction and wear of brake block friction linings are presented in [79]. There are certain characteristic structures on the contact surface in a disk-friction lining type of brake system. They are caused by material wear, which is closely related to the equilibrium of the stream of increase and destruction of firmly stuck "patches" on the contact surface. These patches-clusters of scraps detached from the surface of the block's lining due to abrasion (presented on photographs taken with the use of a microscope)-modify the value of the adhesion surface friction coefficient.

The overall aim of the research was to determine the basic principle of the brake blocks wear and to formulate a new type of differential equation of the second order that would describe the changes of the dynamic friction coefficient. The differential equation proposed by the author describes a short-term stationary behavior during friction of the brake block lining against the brake disk, which seems to be the model's serious limitation.

In this case, engineers attempt to maximize the friction force and minimize the wear in order to achieve appropriately long endurance of the system's frictional elements. Brake block linings usually consist of over 20 different chemical compounds. The chemical constitution of the brake systems' contact surfaces affects the friction processes and thus influences shaping the structural coatings in the contact areas.

Because not all relations between the surface's chemical constitution and the dynamic friction coating are known, extensive research has been made with the use of optical microscopes that are capable of characterizing tribological surfaces on the nanomechanical level [80]. Producers of brake blocks manufacture their own materials for their products. The trial-and-error method is often applied to optimize the chemical constitution of the brake block lining.

As it turns out, friction in brake systems is not sufficiently explained as far as physics and dynamics are concerned. The value of the friction coefficient is usually within the range $(0.1-$ 0.9 ), and it falls as the disk's temperature and the friction force decrease. The value of the friction force in cars is usually $500 \mathrm{~W} / \mathrm{cm}^{2}$, and the temperature in the contact area reaches $300{ }^{\circ} \mathrm{C}$. The effect of decreasing the friction coefficient along with an increase of the friction force is called the "fading effect." It is caused by a nonuniform increase of the thermal force. The results of an experimental analysis prove that rather periodical changes of thermal boundaries occur on brake disks.

Another effect that appears in clutches and brake systems is a periodical change of the dynamic friction coefficient in time. The length of the vibration period changes from a few to a few hundred seconds. The fading and periodical change of the dynamic friction coefficient have been explained in [79] by means of a 


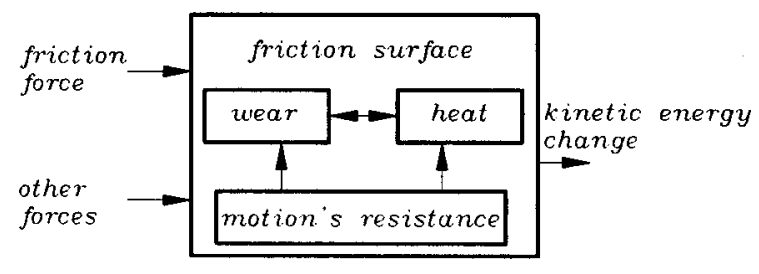

Fig. 15 Basic energy flow in systems with friction

differential equation of the second order. Some theoretical hypotheses derived from the analyses of this equation have been empirically verified.

An energetic analysis of friction has resulted in an observation that a friction force between a rigid body excited by an external force and a flat slip surface should be as large as possible to help maintain the nonzero sliding velocity. Streams of energy, the density of which should be independent of the sliding velocity according to Coulomb, correspond to the contact areas of the brake block lining and the brake disk. The energy's density occurs, physically, in the thermal energy, in the changes that occur on the contact surfaces, and in the material itself. In order to study the elementary friction processes, an analysis of basic energy streams has been made. Figure 15 contains a diagrammatic representation of such streams.

The considerations presented thus far serve as a basis for an assumption that because of the complex dynamics of friction, the friction coefficient $\mu$ ought to be determined with a system of differential equations that describe the energy changes. The considerations on the friction surface structure in the brake block lining adhesion area form foundations for an energetic friction model for brake systems represented by the following system of nonlinear differential equations of the first order:

$$
\begin{gathered}
\dot{\mu}=-a(n v \mu-\eta) \\
\dot{\eta}=-c\left(\eta-\eta_{0}-\alpha n v\right)-\gamma\left(\eta^{4}-\eta_{0}^{4}\right)
\end{gathered}
$$

where $n v$ is a nondimensional parameter dependent on time, which describes the normal force and the tangential force, $\eta$ $=b / a T_{e f}, T_{e f}$ is an effective temperature on the surface on the adhesion area, and $a, b, c, \alpha, \gamma$ are the model's parameters.

Neglecting the small nonlinear terms and assuming $n v$ to be constants, the following stationary solution is obtained:

$$
\begin{gathered}
\eta=\eta_{0}+\alpha n v \\
\mu=\frac{\eta_{0}}{n v}+\alpha
\end{gathered}
$$

Temperature is a linear function of velocity and normal force, whereas friction coefficient is described by the falling (due to the velocity and the normal force) characteristic. Ostermeyer [79] also emphasizes the necessity of taking wear into consideration in modelling brake systems with dry friction.

Empirical observations of fading prove that a leaping decrease of the friction coefficient's value occurs during a leap of the brake disk's rotational speed. The given friction model explains fading effect caused by a smaller increase of the speed of the contact area destruction in comparison to the speed of the development of the strongly fixed "patches."

\section{Dry Friction and Dynamical Systems Theory}

The theory of modeling friction processes described in Sec. 2 embraces a wide range of problems investigated in order to discover physical and dynamic phenomena as well as to determine the mathematical relations that describe them. Many theories have been developed on the grounds of the nonlinear theory that enable to make qualitative or quantitative analyses of the behavior of discontinuous dynamic systems described with the use of various friction models. Therefore, apart from the works that focus on investigation of typical systems with friction, this review paper will include also the literature that describes such well-known problems in the nonlinear vibration theory as: periodic and chaotic vibrations, Poincaré portraits and sections, Lyapunov exponents, and bifurcations of solutions.

The phenomenon of self-excited vibrations caused by friction has been extensively described in the technical literature. A simple oscillator with one degree of freedom [81] was the first to show the occurrence of such vibrations. The necessary condition of the occurrence of the vibrations in that oscillator was a nonzero deflection of the dry friction characteristics given in the form of a relation of the friction force and the relative velocity between the moving belt and the rigid mass that oscillated on it. Hassard et al. also investigated an oscillator of that type [82], but in order to explain the phenomenon of self-excitation of vibrations they chose an approach based on the use of the theory of discontinuous systems' bifurcations.

Engineering practice suggests the necessity of investigating self-excited vibrations with many degrees of freedom. Their occurrence can be observed during paper production processes. When the conveyor belt moves between rotating rolls, certain parts of its surface that differ in brightness of color can be noticed. A thorough investigation has proved that they are caused by selfexcited vibrations of the rolls. Taking the described process into consideration, a qualitative analysis has been made to investigate the rolls' self-excited vibrations caused by dry friction in a system of two degrees of freedom. The analysis has been based on investigation of the changes of the system's total energy derivative (it suggests the occurrence of boundary cycles) and is described in work [83].

Some interesting considerations on self-excited vibrations are presented in work [84], where a mechanical system with four degrees-of-freedom serves as a model of a singular surface contact between two masses. Friction force depends, in this case, on the frictional bodies' relative velocity and on the change of the normal force. The solution of the system of nonlinear ordinary differential equations describing the system's motion can be found with the use of the approximate analytical and numerical methods supported by experiments.

The approximate analytical method forms the foundation for formulation of averaging nonlinear differential equations of the first order. Work [84] uses an example of self-excited vibrations caused by dry friction in a system with two degrees-of-freedom to describe the procedures required in that case. Applying the presented method allows one to analyze the stationary and nonstationary states that occur in the investigated system.

Among various examples of the occurrence of self-excited vibrations there are also high-voltage transmission lines and bridge suspension structures exposed to constant operation of winds, or pipe lines immersed at water reservoir outlets exposed to operation of water currents. Self-excited vibrations may damage or destroy the structure in such situations, as in the case of the Tacoma Narrows Bridge, for instance. In certain conditions, self-excited systems disperse energy in the form of a harmful noise. Sometimes their presence in electronic devices, such as analog-todigital converters, may be desirable and intended, since they enable one to utilize the dynamics related with them to scan the frequencies that characterize the input state $[85,86]$. Deterministic models with static and dynamic dry friction are applied to describe the dynamics of faults and to explain earthquake tectonic processes [87-90] (see also Burridge-Knopoff model presented in Sec. 2.2.1). Self-excited vibrations cause premature wear of cooperating machine parts - as it is presented in [91] that deals with the problem of dry friction occurring during rail vehicles' motion (see also the results of numerical calculations presented in [92]).

Another problematic aspect of mechanical vibrations excited by friction of cutting tools and a processed object may be found in 
[93-96]. The squeaking noise of machine cutting tools (a lathe tool, for example) during machining is an undesirable phenomenon. The accompanying disadvantageous effects, such as noise, increased roughness of the processed surface, and the tool's decreasing strength, accelerating the degradation of the tool's usefulness. The list of numerous coefficients that cause the squeaking noise during machining includes exciting, regenerative and selfexcited vibrations. The self-excited and regenerative vibrations, which depend to a great extent on the relative motion between the tool and the processed object, are especially interesting. The squeaking noise caused by self-excited vibrations during turning may occur as a result of the changes of the friction force on the cutting tool's blade, which, in turn, are caused by a dynamic change of the friction angle and the cutting angle.

The work of Stefański et al. [97] contains a description of a self-excited oscillator with friction designed and constructed for empirical analyses of dry friction effects. A mathematical model is also formulated and the influence of different types of classic friction characteristics on the behavior of a proposed oscillator is investigated numerically.

The phenomenon of friction induced in many physical systems by self-excited and parametric vibrations is analyzed in works [98-100]. If those types of vibrations simultaneously occur in a system then the problem becomes more complex as far as the mathematical notation and the physical interpretation of the system's behavior are concerned. Such types of vibrations occur in mechanical devices, such as a combustion engine, for instance. In certain conditions, self-excited vibrations of a piston and parametric vibrations of a crankshaft can be observed. Self-excited and parametric vibrations caused by dry friction in a three-degree-offreedom system are widely described in [101]. This work analyses a nonlinear parametric system consisting of a rectangular rotor placed on an oscillating rigid base. The areas of parametric instability are identified with the method of power distribution with extension due to two perturbation parameters related to the parametric excitation and the friction coefficient. A mathematical analysis serves to determine the influence of chosen parameters of the systems on the shape and size of the instability areas of the first kind.

The self-excited character of vibrations and the discontinuous form of differential equations enable one to observe stick-slip phenomena in real mechanical systems or in numerically modeled systems. As it turns out, also the relation of friction force and relative velocity in the case of two oscillating bodies leads to the occurrence of nondecaying vibrations in a two-degree-of-freedom autonomous system. The transitions from the slip phase to the stick phase, which occur during the changes of the phase space's dimension (within the range from 4 to 2), play a principal role in the system's dynamic behavior. Works [102-104] are particularly interesting for their examples of vibrations in mechanical systems caused by friction, in which stick-slip phases are observed.

Stick-slip movements that occur in the case of a pendulum with dry friction (also called Coulomb friction) belong to the less complex ones [105]. Works [106,107] describe the scientific research, the aim of which is to prevent stick phases from happening on the friction surface. It is necessary to add that although the study of such systems was initiated by Den Hartog [106] in 1930, it was not continued until the 1950s [107,108]. Den Hartog simplified the problem of vibration with friction and presented a periodical solution (that consisted only of the slip phase; see also [109]) of the oscillator's response with harmonic excitation modeled with Coulomb law. Much later, a similar problem was analyzed with the use of the following techniques: numerical research in various domains of time, research in various phase spaces [106,110,111], an incremental harmonic balance, an equivalent linearization method [112-114], and others.

Den Hartog's work served as the basis for the calculation of periodical solutions (occurring only during the pure slip phase) for the response of an oscillator with Coulomb friction affected by harmonic excitation described in work [109]. The work presents a comparison of Den Hartog's results [81,106] with regard to an additional new calculation in relation to the maximum velocity and its delay time. The basic advantage of the new approach is that a simple relation has been derived to calculate the minimum of the amplitude of the exciting force necessary to provide the pure slip phase between the oscillating body and the adhesion surface. Compatibility of the assumptions and accuracy of the results have also been proved by means of a comparison to the exact solutions [115].

Finding solutions to the systems of differential equations that describe the dynamics of systems with friction often evokes numerical problems, such as impossibility to determine the stick phase correctly or too long a calculation time, for instance. Therefore, work [116] applies the Hénon method [117] to solve a selfexcited dry friction system with two degrees of freedom. Significant shortening of the calculation time and improved accuracy of the phase trajectory with a clearly marked stick phase have been obtained on the basis of conducted analyses.

Work [118] analyzes a self-excited dynamic system with two degrees of freedom with Coulomb friction law. The friction characteristics are approximated by an arctan function. The problem refers to a classic system of two masses located on a moving belt. The belt's equations of motion are brought to a nondimensional form. Many interesting examples of nonlinear dynamics have been discovered regarding the stick-slip phenomenon [22,119].

Carlson and Langer $[89,120]$ and Carlson et al. [121] have investigated the stick-slip effect in multidimensional systems and formulated a theory and conditioning of a mechanism responsible for earthquakelike events and noises. In addition, Vallette and Gollub have investigated stick-slip vibrations that occur in a space-instantaneous system of an extended membrane contacting a glass rod. By means of experiments and the use of the wave propagation method the stick-slip behavior has been presented.

Work [122] and two review articles [123,124] contain some interesting formulations and a theory concerning the stick-slip phenomenon. According to an observation, basic difficulties that appear during the description of this phenomenon result from the nature of friction law, which changes the direction of the friction force during the change of the relative velocity's sign. Observations conducted by Oden [123] and Brommundt [124] have proved that an occurrence of the stick-slip motion in a model system does not require the assumption of the difference between the static and the dynamic friction coefficient. Work [125] utilizes that conclusion and assumes a singular value of the friction coefficient in relation to the relative velocity (in the form of an sgn function). In a mechanical system consisting of two masses connected by a spring and oscillating perpendicularly in a cylindrical pipe, a nonsmooth Coulomb friction characteristic has been applied.

Undoubtedly, friction, wear, heat emission, or deformations caused by a temperature increase are complex processes that require a special approach. Awrejcewicz and Pyryev [11] have investigated a nonlinear problem of a thermoelastic contact of a rotating shaft and a rigid bush fixed by springs onto the base. In their research they have applied Laplace transform and the perturbation method and have assumed that the friction coefficient is a nonlinear function of the relative velocity. The problem has been reduced to a nonlinear system of differential and integral equations. The numerical analysis has resulted in the observation of self-excited vibrations during the stick-slip movements and the areas of stability of a stationary solution.

The dynamics of an oscillator with two degrees of freedom with dry friction is investigated in [126]. The system consists of a mass shaped as a channel bar oscillating on a base that moves at a constant velocity, and another mass (supported by a spring) located inside the channel bar and capable of perpendicular movements. A constant friction coefficient is assumed on the contact between the channel bar and the base's surface. Solutions that lead 
to obtaining boundary cycles during subsequent stick-slip phases are described. The results of numerical analyses lead to a conclusion that one of the reasons for the occurrence of the stick-slip phase in the system is the channel bar shift coupling tangential to the friction plane with the oscillating mass' perpendicular motion.

The unstable nature of vibrations that reveals during stick-slip motion has been observed in Van der Pol oscillators, in which the friction coefficient decreases along with an increase of the relative velocity $[14,15,126]$. It also happens in the case of oscillators with an additional spring, which is fixed onto the oscillating mass, that causes a change of the pressure in the normal direction toward the friction surface [123]. Experiments justify considering the elasticity that influences the change of the perpendicular pressure onto the friction surface. Conducted observations confirm the assumptions that the mass' relocations in a perpendicular direction toward the contact surface occur during the slip phase [127].

Among many mechanical devices there are ones that are equipped with an additional system with a disk, which serves to generate an intended effect of friction. The most popular are car brake blocks, computer hard disks, or circular saw machines. The study of dynamic instability of such mechanisms described in [128-131] refer to an analysis of stationary disks excited by a rotating loading and a disk affected by constant loading [128]. In all the cases mentioned above, the investigated system's transition into unstable states occurs at certain specific values of parameters (of mass, rigidity, and damping).

Friction modeling by description, with regard to progressive (following) force, is widely known in the literature on disk vibrations. Work [132] investigates friction with regard to following force, which appears in disk drives of personal computers. Interestingly enough, the results confirm the fact that the vibrations propagate in the direction that corresponds to the occurrence of instability observed on the disk's surface.

The occurrence of vibrations during an elevators' motion belongs to the less thoroughly investigated problems. Miwa [133] and Sissala et al. [134] used a simple mass-elastodamping system to model an elevator's drive system with a drive wheel. Continuing their study, Wee et al. [135] investigated nonlinear, velocitydependent, stick-slip vibrations that occur during a sliding-metal contact. The nonlinear behavior of that kind (observed during an elevators' motion) is characterized by the occurrence of discontinuity on the adhesion surface between the wheel and the guide rail. The described analysis aims at showing the effects of nonlinear dynamics on the contact of the studied elements, suggesting a mathematical model with a two-step evaluation of its parameters and suggesting a method of determining them (on the basis of computing techniques).

The stick-slip motion is unstable because of the slip phase caused by a decrease of the friction force value along with an increase of the relative velocity in a susceptible mechanical system. Works [136-141] explain that process thoroughly. Stick-slip vibrations are usually explained, starting from the initial state in the stick phase, and then the study of the entire phase stability is carried, including verification and calculations for subsequent phases that follow within a long period of time [142-152]. It is also necessary to mention work [90], which, as one of a few, includes the study of the consequences of starting stick-slip motion from the point in space that corresponds to the phase of a "weak" slide. That case has been developed into a problem of determining the time instant or the point in the phase space in which the stick-slip motion can be eliminated from a mechanical system through an increase of velocity beyond its critical value and then a decrease back to its initial value [153].

The problem of stabilization of nonsmooth systems is illustrated and discussed $[154,155]$. An importance of the contact and impact phenomena in many mechanical systems are discussed in Refs. [156,157] (grinding and deburing problems in manipulators performing tasks), [158] (filamentary brushing tools for surface finishing), and [159] (robotic systems).
In general, collisions associated with friction and impact are considered as a harmful behavior, but in some cases impacts are provoked intentionally $[160,161]$ in order to dissipate energy and contribute toward stabilization of the considered system. The accurate conditions for various types of stability properties of the closed-loop system involving a free-motion phase, a permanently constraint phase, and a transition phase are formulated by [162]. In addition, the existence of a specific transition between permanent constraint phases and free-motion phases is rigorously proved.

The analysis of the research described in work [163] and further developed in work [164] shows that friction that causes selfexcitation of tangential vibrations in the stick-slip motion may be the reason for a sudden significant increase of the acceleration value and the vibration amplitude. According to observations, probability of an occurrence of a stick-slip phase during deceleration is significantly bigger because of the lengthening of the phase that corresponds to the decrease of the acceleration value and to the tangential increase of the system's rigidity.

Brace and Byerlee [165] initiated experimental research on the analogy between subsequent seismic events and stick-slip motion. Numerous critical analyses of the mechanisms for seismic slide of blocks of rock consequently appeared. Some of them referred rather to the analysis of the slip phase (with the use of a discontinuous change of the sliding velocity step) before the occurrence of instability. Moreover, the physical aspect of normal relocation in the slip phase was not fully explained. Experiments proved that vibrations perpendicular to the contact surface occur (for various materials) during the stick-slip motion. Direct measurements of metals in an experimental stand proved that change in electric conductivity of the contact surface with stick-slip vibrations is caused by normal vibrations [166]. Similar vibrations were also observed during an investigation of rubber foam. A photography technology applied in that case helped one to observe relocations of light-emitting diodes (LED) placed a few centimeters below and above the slide surface $[167,168]$.

During the experimental analysis with the use of LEDs described in work [169], a peculiar behavior of two rubber foam masses was observed: within certain time intervals in the slip phase they were losing adhesion. The results of the presented investigation may lead to a conclusion that a decrease of a pressure component in the normal direction in the slip phase is caused not only by perpendicular vibrations, but also by total loss of adhesion between contact surfaces. It means that the value of pressure in the normal direction tends to zero. The stick-slip phenomenon occurred only when pressure was very low, yet it could not be fully explained when the contact surfaces separate during heavy pressures in the adhesion area and when the slip surface roughness influences the system's dynamics (see also, the results of numerical analyses in works [170-173]).

Work [174] includes the research results that describe the phase of adhesion loss in stick-slip motion within a vast range of pressure values perpendicular to the adhesion surfaces of investigated polymethacrylic samples. The analysis described in this work constitutes a part of extensive experimental research $[175,176]$ focused on determining parameters and describing how normal pressure on the adhesion surface, loading velocity, and roughness influences the stable slide in stick-slip motions.

In order to comprehend the changes of friction force causing stick-slip motions, the relation between friction force and velocity in the slip phase has been determined through empirical measurements (see Sec. 2.2.1.2 on experimental static friction models and also works $[144,145,177-185])$. The authors of the quoted works are preoccupied mostly on determining a loop that would not intersect on the surface in the friction force-relocation relative velocity coordinates. The results of the investigation do not bring any sufficient explanation for the mechanism of such a type of friction curve shape. Reference [186] shows that the shape of an intersecting or a nonintersecting loop on the surface in the friction 
force-relative velocity coordinates may be obtained through changing the rigidity of a spring tangential to the friction surface of the investigated frictional pair. Moreover, the occurrence of the intersecting loop in the investigated system of coordinates is not produced by a real physical phenomenon, but it is a result of the vibrations' "neglecting" some velocity changes, which happens for most cases of rigidly connected and sliding bodies.

Investigation of the adhesion area during friction is a complex process because it depends on many physical, mechanical, and material parameters. It is also dependent on more or less complex relations that cause difficulties in formulating a mathematical description and understanding the essence of the problem (see also Sec. 2.2.1.3 on the mathematical approach to modeling friction and works $[187,188])$. In order to learn about the conditions on the adhesion surface during dry friction motion, which cause changes in the friction force, several theories based on various hypotheses have been developed. The basic conclusions include the following: (i) for a state in which the adhesion surface has a constant quantity and for which microrelocations remain in a linear relation with a force tangential to that surface, the dynamic friction coefficient depends on the stationary adhesion (sticking) time $[189,190]$ (this phenomenon explains the connections made on the adhesion plane, which in time become stronger); (ii) during the slip-phase, the dynamic friction coefficient is assumed in many theories (see also Sec. 2.2.1 on dry friction static models) as a function of the relative sliding velocity [191,192].

Reference [193] contains an analysis of dry friction occurring at small relocations $(\sim 50 \mu \mathrm{m})$ on the adhesion surface and at almost zero sliding velocity (kept below $0.2 \mu \mathrm{m} / \mathrm{s}$; see also Sec. 2.2.1.5 where a dry friction model on the atomic scale is described). Because of those conditions the quoted hypotheses and the standard approach theory could not have been applied in that case and the work describes the experiments conducted in order to explain the above-mentioned aspect of dry friction. The results of the experiments show that the dynamic friction coefficient value increases after the conditions of the stationary adhesion on the investigated surface have been satisfied, and it decreases along with an increase of the slide length during the microslide. The changes measured on the adhesion surface are continuous and depend on the relocation within asymptotic boundaries.

An oscillating system, excited by a stream of air and made of a mass, connected elastically with a stable base and a pendulum, is analyzed in Ref. [194]. Continuing the previously mentioned research, the authors additionally included (in [195]) the conditions of dry friction in the connection of the mass and the base, which significantly affected the system's dynamics. With the use of the mathematical analysis supported by numerical calculations they showed that the trivial solution of the motion's system of equations is stable, whereas the semi-trivial solution (in case of a motionless pendulum) is unstable within the entire range of the airstream velocity values.

Work [92] presents an analysis of a linear dynamic system with damping connected with a nonlinear one-degree-of-freedom system, in which adhesion conditions and Lagrange multipliers have been applied. The dampers that utilize dry friction for functioning are assembled into a nonlinear elastic system in the form of a bar and also determine its fields of asymptotic stability.

The mathematical approach to nonlinear physical phenomena involves many difficulties connected, among many other things, to the fact that they are described by differential equations, the right sides of which are discontinuous and sometimes nondifferentiable. The book by Kunze [44] contains an extensive analysis of mathematical aspects of discontinuous dynamic systems with friction and impacts and also describes mathematical methods applied by engineers during experiments. The author devotes attention to the problem of dry friction leading to differential inclusions, often called multivalued differential equations [42,105,196] (see also Sec. 2.2.1.3). Furthermore, he investigates the problem of almostperiodical solutions [197] and methods of calculating Lapunov exponents for a pendulum with dry friction. The work also contains a formulation of the relation between the dynamic parameters of discontinuous systems with friction and impacts [198].

One of possible perspectives devoted to nonsmooth system dynamics is addressed by Georgiadis et al. [199], where shock isolation designs based on nonlinear energy pumping caused by nonsmooth stiffness elements are studied. The term energy pumping is understood in a sense of the nonreversible transfer of vibration energy from its point of generation to a predetermined spatial area (the nonlinear energy sink), where the vibration localizes and dissipates. In contrast to classical linear vibration absorber, it is shown that the nonlinear energy sinks are capable of efficiently absorbing energies caused by transient broadband disturbances.

The rigorous approach to dry friction problems is presented by Fečkan [200], where the existence of a continuum of many chaotic solutions for certain differential inclusions, i.e., small nonautonomous multivalued perturbations of ordinary differential equations possessing homoclinic solutions to hyperbolic fixed points are shown.

References [201,202] focus on uniqueness of solutions of the motion equations for a mechanical system with dry friction. The equations refer to a general case. These works quote several definitions applied to formulate (and subsequently utilize) the theorems on the existence and uniqueness of solutions.

Vibrations described with a mathematical model consist of a stick-phase with a relatively large static-friction coefficient, and a slip-phase, in which the coefficient-also called a dynamic friction coefficient-is considerably smaller. That is why the systems with dry friction are sometimes characterized by static behavior and more often by various dynamic behaviors represented by periodical, quasi-periodical and chaotic movements [127,203-205].

The works by Lorenz [206] and Ueda [207] gave beginning to the observations of chaotic movements in simple discrete nonlinear systems described by differential equations of at least the third order. Until today many works (for example, [208-210]) dealing with chaotic movements occurrence in physical systems have been published.

The plane dynamics of a rigid block simply supported on a harmonically moving rigid ground exhibiting unilateral contacts, Coulomb friction and impacts has been studied by Ageno and Sinopoli [211].

The results that enable one to observe the stick and slip phases in chaotic dynamics of simple dynamic systems with one degree of freedom with friction are presented in work [212]. Its authors focus on numerical and analytical analyses of the search for chaos during the stick or slip phase. Melnikov's method applied to the investigations of discontinuous dynamic systems with dry friction is a main topic of work [213]. The work examines the problem of the search for chaos during a slightly forced stick-slip motion in a dynamic system. A stick-slip chaos analytical prediction has been confirmed analytically.

Two-dimensional maps for differential equations (of the second order, for instance) can be made in a simple way using the Poincaré section. Therefore, such a simple mapping can be made for investigations of an extensive class of dynamic systems modeled by nonlinear oscillators [214-217]. Such a mapping may also serve as a useful tool for explaining sudden leaps of a phase trajectory. In real systems, trajectories are attracted by other attractors exactly after a leap, which, consequently, is the main reason for bringing an attractor to infinity [42,218,219].

Calculating Lapunov exponents belongs to one of the most fundamental issues related to a quantitative analysis of dynamic systems. The theory developed by Oseledec [220] and the numerical algorithms derived by Benettin et al. [221,222] and Wolf et al. [223] enable one to estimate the spectrum of Lapunov exponents for the systems described by continuous equations of motion. Moreover, if the equations of motion are unknown or are pre- 
sented in a discontinuous form, then other methods are applied, such as the ones based on the reconstruction of an attractor from a time series [223], for instance.

There is a growing tendency to consider real mechanical systems with friction or impacts as discontinuous dynamic systems. Numerous works on the theory of dynamic behavior of nonlinear systems with friction have appeared recently (see [220-223]). Nevertheless, only a few of them [92,223,224], for instance, contain a genuinely innovatory approach to calculation of Lapunov exponents for that type of systems.

Synchronization of chaos [225] has been applied in [226,227] to present a method of calculating largest Lapunov exponent for a system with friction and impacts, which is based on investigations conducted by Fujisaka and Yamada [228]. They found a nonlinear relation between the value of a coupling coefficient (corresponding to the synchronization between two identically examined systems) and the value of the largest Lapunov exponent. The synchronization condition was formulated only for negative symmetric back couplings between the analyzed systems.

An exact solution of a discontinuous system of differential equations (e.g., describing a mechanism with dry friction) sometimes requires the use of sophisticated methods that enable one to determine the points of the real movement trajectory on the phase plane regarding all peculiarities (see also. Sec. 2.2.1.3 and works $[42,44,105,196])$, such as the stick-slip transition, for instance. Therefore, works such as [229] present solutions of the system of differential equations that describe the motion of a two-degree-offreedom system with friction derived with the use of the "exact" Hénon method [117]. In order to achieve constant time intervals between the trajectory's points, the obtained trajectory is interpolated and Lapunov exponents are calculated from a time series.

\section{Friction Advantages: A Brake Mechanism}

Presently, when computerization and technology are highly developed, the meaning of principal branches of science has been increasing significantly. Modern technology requires high-speed functioning or unerring precision of machines (for example: heavy machines, such as cranes, traveling bridges, etc., as well as manipulators or robots) in varied environments. Therefore, accurate modeling of a great number of dynamic phenomena that occur in machine systems has become a necessity.

The present level of numerical methods' development and the progress in highly advanced computerization enable one to choose adequate physical and mathematical models. Yet, the overall purpose is not to formulate a real and general description of a given phenomenon at any cost, but to reflect its "nature" in a specific regime of the investigated system. Moreover, the well-known and traditionally described phenomena often require remodeling based on new achievements of principal sciences and enhanced computing methods. Analyses of such a complex phenomenon as friction include all aspects of the above-mentioned approaches.

Friction has been an object of interest of many branches of science: mechanics [30,230], tribology [231,232], mass and heat transfer [233-235], the theory of elasticity and plasticity, materials science [236-238], fluid dynamics [239], intermolecular connections physics, or even physical-chemical processes [240,241] (e.g., corrosion or frictional materials work in varied environments involving the possibility of chemical reactions [242,243]). In general, friction is accompanied by a number of other phenomena, such as stresses, material wear, heat emission, etc. In addition, friction (along with impacts) belongs to the group of processes that require precise mathematical determining $[21,23,44,244-248]$.

It is extremely difficult (perhaps even impossible) to build a general friction model including all possible accompanying processes. Moreover, it seems to be pointless, since only some of the above-mentioned processes dominate in a specific object of study. The difficulties involved with explaining numerous effects of friction that appear during confrontations of mechanical (geometric),

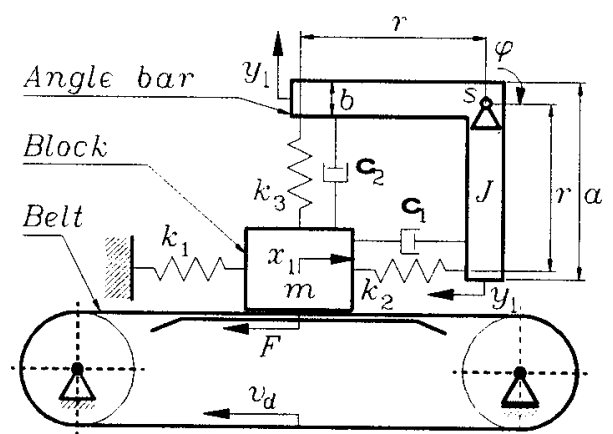

Fig. 16 Theoretical 2-DOF model of a brake mechanism

molecular (adhesive), mechanic-molecular, and energy theories with experiments created the need to model friction with the use of simple dynamic systems for analysis of friction-induced processes [249].

Diverse characteristics of friction can be observed in real dynamic systems. Many models do not require taking friction into account, although numerous systems are based on utilizing friction; hence, omitting the friction-induced effects is impossible. Friction reveals its dominating nature in friction clutches, belt drive systems, as well as in the brake systems in which friction force between a wheel's brake drum and brake blocks causes braking of a vehicle [250,251].

4.1 Engineering Approach. The most widely applied brakes are the ones that are assembled in the wheels of vehicles with certain brake mechanisms.

Let us examine a theoretical brake mechanism illustrated in Fig. 16. It shows that the model is constructed in such a way so that the direction of friction force $F$ is opposite to the force incoming from springs acting beside mass $m$ on the belt. Obviously, when the force in spring $k_{1}$ is larger than friction force $F$, then a loss of adhesion occurs and mass $m$ moves in direction $-x$. At that time, spring $k_{l}$ expands and if its length exceeds its free length, then the perpendicular arm of the angle bar is "pulled" in direction $-x$ and the angle bar is turned in direction $-\phi$. The horizontal arm of the angle bar expands spring $k_{2}$, which decreases the pressure force exerted by the spring on mass $m$ oscillating on the belt. The coupling repeats in the system throughout the entire friction process as long as the belt's linear velocity $v_{d}$ is not equal to zero.

The fundamental element that needs utmost attention while estimating the similarity of the described system (the model) to a brake mechanism (a real object) is the coupling of the mass $m$ transfer on the belt induced by friction (Fig. 16) with the normal pressure force affecting the belt. A model of a brake mechanism with intensified braking force is shown in Fig. 17.

As it is shown in Fig. 17, when the element that initiates brak-
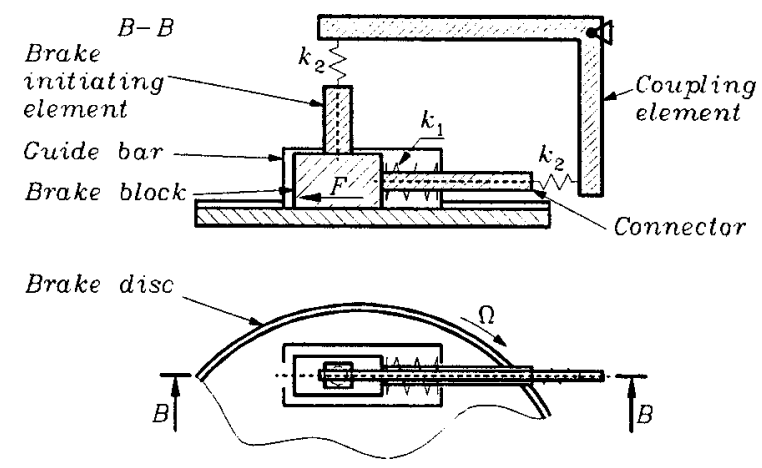

Fig. 17 Model of a brake mechanism with intensified braking force 


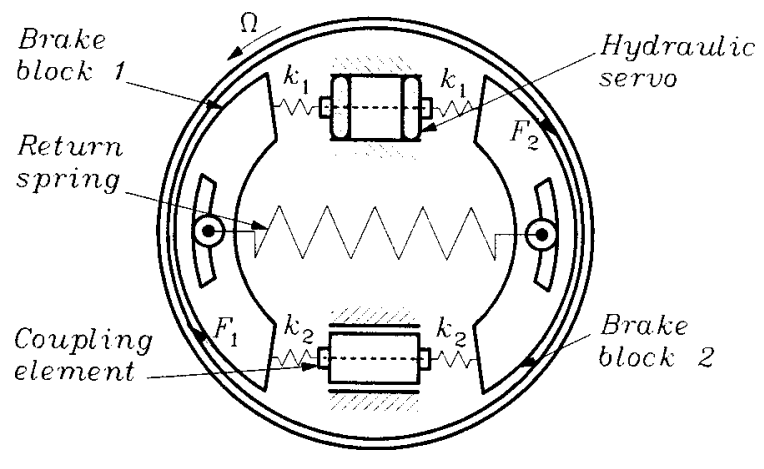

Fig. 18 A scheme of the Girling duo-servobrake mechanism

ing (the pressure on the brake pedal) starts to press the brake block against the disk, then friction force occurs between the block and the disk of a wheel. In effect, the brake block moves in direction $x$, and the coupling element moves or turns and, by an increase of the pressure force, intensifies braking. When the initial pressure decreases (the release of the brake lever), friction force between the brake block lining and the brake disk also decreases and the return spring $k_{1}$ may pull the brake block back to its initial position. It should be noted that coupling of the friction force with the pressure force may function as power-assistance to the braking system. That is why the pressure force on the brake lever may be significantly lowered during braking. The described mechanical coupling can also be obtained with the use of a hydraulic oil system with a pump.

The brake block lining has low susceptibility and can be modeled with a belt when the experiment's conditions are satisfied (see Fig. 16). Additionally, it is possible to choose the materials, of which the brake disk and mass $m$ are made.

According to the conducted investigation, there is a similarity between functioning of the dynamic systems presented in Figs. 16 and 17. The friction model that is examined, assumedly for the system in Fig. 16, approximately corresponds to the brake block lining's friction against a car brake drum (or a disk). Assuming that the same materials are chosen, the model enables one to investigate the phenomena that result from friction and wear of sliding surfaces.

The method of friction modeling that includes the relation between the friction force and the sliding bodies' relative velocity, and between the friction force and the changes of the normal force (the pressure force of the frictional pairs) may also be applied to the study of friction-induced dynamic phenomena in the braking system presented in Fig. 18.

The brake mechanism diagrammatically shown in Fig. 18 is assembled in a popular type of Girling brake, i.e., "duo-servo" (see Fig. 19). When the hydraulic servo initiates braking, the brake blocks 1 and 2 are drawn aside and pressed against the inner surface of the drum. As a result, friction forces $F_{1}$ and $F_{2}$ are exerted between the blocks' linings and the drum and the wheel stops.

A careful analysis of the mechanism shown in Fig. 18 reveals a certain type of coupling. Brake block 1 (also called a "backward" block) takes over a larger part of the friction force at the initial stage of braking, whereas brake block 2 (called a "concurrent" block) impedes with a weaker force. However, the coupling element (the angle bar in the system in Fig. 16) combines the circumferential motion of brake block 1 with the motion of brake block 2 and the pressure force of the latter on the drum's inner surface increases.

The ratio of the braking forces exerted by brake blocks 1 and 2 is about $2: 4$. Brake blocks 1 and 2 are connected by the return spring in such a way that enables them to return to their initial position as soon as the braking process is over. In practice, there are several types of braking mechanisms that function in a similar

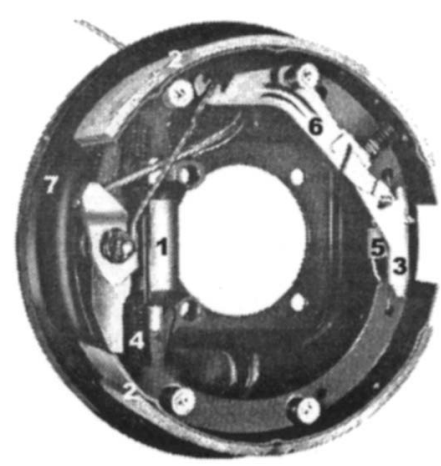

Fig. 19 Girling duo-servo brake: 1-hydraulic servo, 2-brake blocks with linings, 3-coupling element (see Fig. 18), 4-long return spring that pulls the brake blocks back, 5-short return spring, 6-hand-brake mechanism, 7-drum

way (Fig. 19).

The purpose of the considerations presented above is to show that a simple self-excited system (Fig. 16) with a changeable pressure force on the belt may function as a starting point for analyses of friction in brake systems represented by drum brakes.

4.2 Modeled System. The study and prevention of selfexcited vibration of systems with friction is very important in industry, and there is a need of friction pair modeling, which could correctly describe dynamic and static friction forces change between two moveable surfaces. A further-developed model can govern the dynamics of the Girling duo-servo brake mechanism [252] described in Sec. 4.1. Therefore, the schematically illustrated [in Fig. 16] two degrees-of-freedom dynamical system is numerically analyzed and experimentally investigated.

The self-excited system presented in Fig. 16 is almost equivalent to a real experimental rig in which block mass $m$ is moving on the belt in $x_{1}$ direction, and where the angle body represented by moment mass of inertia $J$ is rotating around point $s$ with respect to direction of angle $\phi$. The analyzed system consists of the following parts: two bodies are coupled by linear springs $k_{2}$ and $k_{3}$; block on the belt is additionally coupled to a fixed base using a linear spring $k_{1}$; the angle body is excited only by spring forces; there are no extra mechanical actuators; rotational motion of the angle body is damped using virtual actuators characterizing air resistance marked by constants $c_{1}$ and $c_{2}$; damping of the block is neglected; it is assumed that angle of rotation $\phi$ of the angle body is small, within interval $[+5,-5] \mathrm{deg}$ (in this case a rotation is equivalent to linear displacement $y_{1}$ of legs $a$ of the angle body); the belt is moving with constant velocity $v_{b}$; and there is no deformation of the belt in a contact zone.

Nondimensional equations governing dynamics of our investigated system have the following form:

$$
\begin{gathered}
\dot{x}_{1}=x_{2} \\
\dot{x}_{2}=-x_{1}-\alpha_{1}^{-1}\left[\eta_{1}\left(x_{2}+y_{2}\right)-y_{1}-F\right] \\
\dot{y}_{1}=y_{2} \\
\dot{y}_{2}=\alpha_{2}^{-1}\left(-\beta_{3} y_{1}-\eta_{12} y_{2}-x_{1}-\eta_{1} x_{2}\right)
\end{gathered}
$$

where $x_{2}$ and $y_{2}$ are velocities of the block and angle body, respectively; $v_{r}=x_{2}-v_{d}$ is a relative velocity between bodies of the investigated system:

$$
\alpha_{1}=\frac{\omega^{2} m}{k_{2}}, \quad \alpha_{2}=\frac{\omega^{2} J}{k_{2} r^{2}}, \quad \beta_{1}=\frac{k_{1}+k_{2}}{k_{2}}, \quad \beta_{2}=\frac{\mu_{0} k_{3}}{k_{2}}
$$




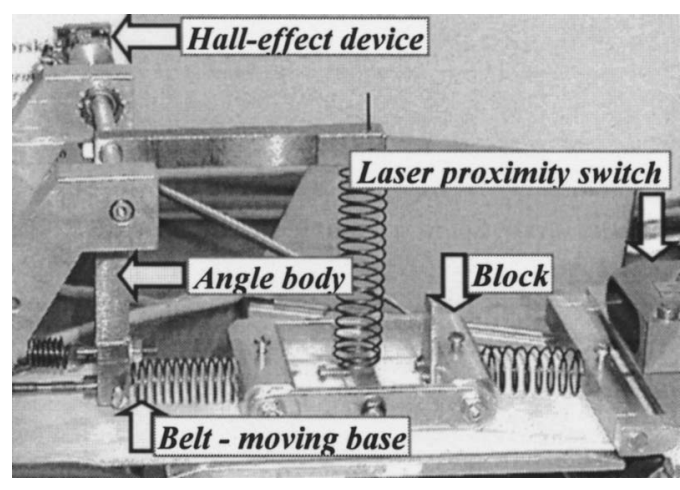

Fig. 20 The laboratory rig

$$
\begin{gathered}
\beta_{3}=\frac{k_{2}+k_{3}}{k_{2}}, \quad \eta_{12}=\frac{\omega\left(c_{1}+c_{2}\right)}{k_{2}}, \quad \eta_{2}=\frac{c_{2} \omega \mu_{0}}{k_{2}} \\
\eta_{1}=\frac{c_{1} \omega}{k_{2}}
\end{gathered}
$$

are the remaining parameters; and $\omega$ is a natural frequency associated with mass $m$. Friction force is described in the following manner:

$$
F= \begin{cases}\operatorname{sgn}\left(v_{r}\right) F_{+} & \text {if } v_{r}>0 \\ \operatorname{sgn}\left(v_{r}\right) F_{-} & \text {if } v_{r}<0 \\ \left|F_{S}\right| & \text { if } v_{r}=0\end{cases}
$$

$F_{+}$and $F_{-}$friction force characteristics are described by linearly and exponentially decaying functions, respectively.

4.3 Experimental Investigations. In this section, the laboratory rig [252-254] designed for observations and experimental research of friction effects, including friction force measurement, is described. Photos of the rig are presented in Fig. 20.

The general view, component parts, and some connectors, such as coil springs, correspond (see Fig. 20) to those schematically indicated elements presented in Fig. 16.

Displacement of the block and angle of rotation are measured using a laser proximity switch and Hall-effect device, which guaranty a nonsticking method of the measurement. Both of them provide linear dependency of the measured quantity versus analog voltage output. Measurement instruments connected through a PCI computer card to LabView software allows one to perform dynamic acquisition of the two measured signals. Disturbances of whole construction, noise in electrical circuits, and other additional maintenances have significant influence on accuracy of any measured signals. Therefore, some signals are filtered digitally (elliptic topology) and a real differentiation preventing high-peaks formation is applied.

Appropriate transformed equations of motion (see: Eq. (20)) can be used for friction force calculation after real-time measurement of state variables of the investigated system. Characteristics of friction force versus relative velocity between belt and block for positive and negative velocities of the belt are shown in Fig. 21.

One may observe that zones occupied by closed functions of the friction model differ significantly. It is a regularity, since angle body causes reinforcement of friction force for positive velocity of moving. Owing to these considerations, $F_{+}$and $F_{-}$friction force characteristics are described by linearly and exponentially (of second order) decaying functions, respectively.

In the case of $F_{+}$branch, the equation of friction-force dependence describing a friction-force model for positive relative velocity has the following form:

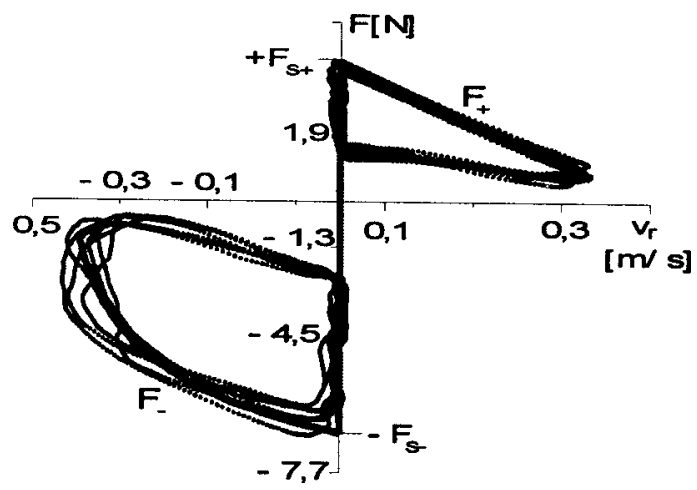

Fig. 21 Friction force characteristics for positive $\left(F_{+}\right)$and negative $\left(F_{-}\right)$relative velocity

$$
F_{+}=F_{s+}-\left|v_{r}\right| \frac{F_{s}-F_{\text {min }}}{v_{r, \max }}
$$

where $F_{s+}$ is a static friction force, $v_{r, \max }$ is a maximum positive relative velocity. The $F_{-}$branch can be described by a secondorder exponentially decaying function describing a friction-force model for negative relative velocity of the following form:

$$
F_{-}=F_{s-}+a_{1} \exp \left(-\frac{\left|v_{r}\right|-v_{r, \text { min }}}{t_{1}}\right)+a_{2} \exp \left(-\frac{\left|v_{r}\right|-v_{r, \min }}{t_{2}}\right)
$$

where $v_{r, \text { min }}$ is a maximum negative velocity and $a_{1}, a_{2}, t_{1}, t_{2}$ are the constant values. The main multivalued function describing friction force changes occurring in our investigated 2-DOF system with a variable normal force is determined from Eq. (21).

4.4 Numerical Analysis: Bifurcations. The friction-force model given by the two-valued equation (21) is transformed to the nondimensional one, and then a numerical analysis based on $F_{+}$ and $F_{-}$friction force characteristics is carried out. Parameters of both models are obtained by both measurement and identification: $F_{s}=3.63, F_{\min }=0.86, v_{r, \max }=0.27\left(F_{+}\right.$model $) ; F_{s+}=F_{s-}=-5.94$, $F_{\text {min }}=-1.42, v_{r, \max }=0.28, a_{1},=3.23453, a_{2}=2.87362, t_{1}=0.0342$, and $t_{2}=0.30529$ ( $F_{-}$model). Numerical analysis with implementation of introduced friction-force dependency has yielded the results presented in Figs. 22 and 23.

The bifurcation diagrams are constructed by changing a parameter in the interval $(0.2,0.7)$ with the step 0.001 , from which we get 500 Poincaré maps. Then, one of the phase axes is taken and all results are presented versus a parameter. Another way is that for increasing the parameter values to change the initial conditions, and contrary to the first case, to leave an attractor (in the previous case, the solution did not leave the attractor).

An example of an bifurcation diagram is shown in Fig. 22. Beginning from the smallest considered values of $\alpha_{1}$ we observe one-periodic motion, but for $\alpha_{1} \approx 3.1$ period tripled bifurcation with an increase of $\alpha_{1}$ occurs. In the vicinity of bifurcation point $\alpha_{1} \approx 4.0$, the period tripled bifurcation with a decrease of the bifurcation parameter is observed once again. It should be emphasized that for a large interval of changes of the bifurcation parameter, only periodic motion can be reached by our analyzed system.

An interesting example of more complex bifurcations is showed in Fig. 23. One may trace how the successive period doubling (accompanying a decrease of $\alpha_{1}$ parameter) leads to a chaotic motion, which exists for $\alpha_{1} \approx 2.9$. Additionally, period- $n$ windows for $\alpha_{1} \approx(3.15,3.05,2.95)$ are reported.

\section{Conclusions}

The classical and modern approaches of friction phenomena in various mechanical objects have been presented. It was empha- 


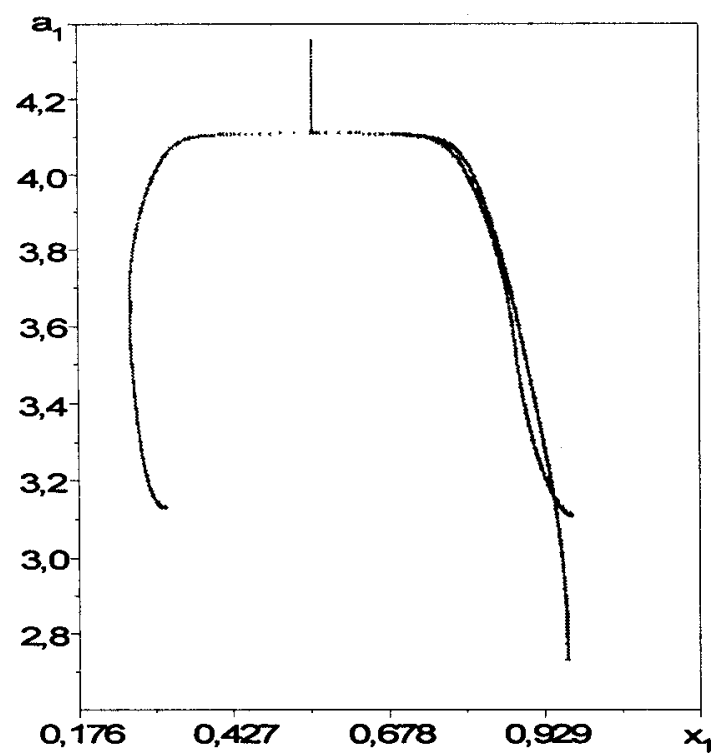

Fig. 22 Bifurcation diagram of $\alpha_{1} \in(2.72 ; 4.32)$ parameter versus $x_{1}$ displacement in the time interval from $\tau_{0}=1000$ to $\tau_{k}$ $=51000 \quad\left[\alpha_{1}=3, \alpha_{2}=1.159, \eta_{1,2,12}=0, \beta_{2}=0.577, \beta_{3}=1.825, \gamma_{1}\right.$ $=0.2, \gamma_{2}=0.8, v_{d}=0.6, \mu_{0}=0.7$, and initial conditions: $x_{1,2}(0)$ $\left.=y_{1,2}(0)=0\right]$

sized that foundations for present studies of difficulties connected with nonlinear dynamics are provided by the well-known mechanical theories.

Friction laws described by a slip-velocity-dependent coefficient were introduced to model the nonsmooth stick-slip phenomenon. The friction mechanism yields self-sustained vibrations in mechanical systems with dry friction. The dynamic aspect seems to be more important in the behavior of such systems. Some of theoretically and experimentally obtained friction force or coefficient characteristics tend to prove that their implementation depends especially on the type of engineering applications.

Owning to complexity of multidimensional systems with nonlinearities associated to dry friction, the unilateral contacts can

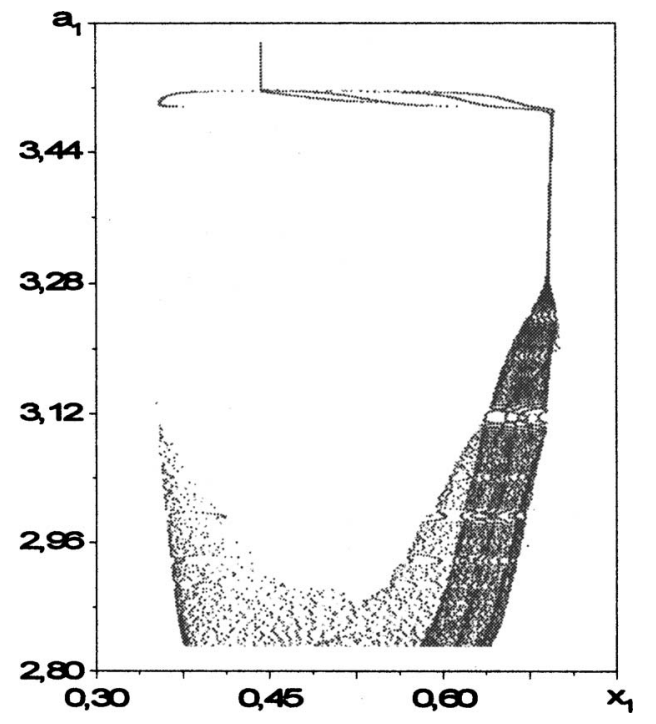

Fig. 23 Bifurcation diagram of $\alpha_{1} \in(2.83 ; 3.58)$ parameter versus $x_{1}$ displacement for $\alpha_{2}=1.093, \eta_{1,2,12}=0, \gamma_{1}=0.152, \gamma_{2}$ $=0.609, v_{d}=0.1, \mu_{0}=1.2, \beta_{2}=1.729, \beta_{3}=2.441$, and initial conditions: $\tau_{0}=1000, \tau_{k}=51000, x_{1}(0)=0, x_{2}(0)=0.1, y_{1,2}(0)=0$ develop instabilities even if the Coulomb friction law is chosen. Therefore, the law with a slip-velocity-dependent coefficient is still used as a good approximation of a stick-slip motion description.

This review presents comprehensive knowledge about some interesting dynamic nonlinear behaviors that were reported by many researchers in systems with impacts and friction. The particular systems' analysis, including complex bifurcations and chaotic motions, has also been briefly presented.

The first approach is concerned with computer programing, and after this the real object recognized by a laboratory rig is assembled and then investigated as well. These actually performed simulations are applied to verify the results of measurements with the use of numerical analysis.

Engineering investigations and a numerical approach have allowed the determination of the dry friction force model presented in Sec. 4. Two nonsymmetric branches of the friction law confirm that some tri-bological effects in the contact zone are more complex and therefore difficult to describe. It is worth noting, that the experimental characteristic represented by the $F_{-}$model is as a first approach recommended to be used during analysis of friction effects occurring in systems, where the normal force acting between cooperated surfaces varies in time. From the point of view of practical application of the experimentally determined friction law for changes in normal force is that the nonlinear $F_{-}$model, especially, can be applied to investigate affected-by-friction tribological effects occurring between brake blocks and the brake disk. An idea for the friction pair modeling using both laboratory equipment and numerical simulation is proposed, allowing observation and control of friction force.

Summing up, there is still visible a need to understand nonsmooth dynamics of mechanisms and to allow the possibility for scientists to estimate effect of its occurrence.

\section{Acknowledgment}

This work has been supported by the Polish State Committee for Scientific Research (grant No. 4 T07A 031 28) and the Department of Mathematics of the Central European University in Budapest.

\section{Nomenclature}

$v_{r}=$ relative velocity

$v_{d}=$ velocity of the base

$F=$ friction force

$F_{s}=$ static friction force

$F_{k}=$ dynamic friction force

$F_{C}=$ Coulomb friction force

$F_{+}, F_{-}=$friction force for positive and negative relative velocity

$F_{g}=$ gravity force

$F_{u}=$ arbitrary directed friction force

$N=$ normal force

$T=$ torque

$P=$ pressure

$x_{i}, y_{i}=i$ th displacement

$\dot{x}_{i}, v_{i}=i$ th velocity

$\ddot{x}, \dot{v}=$ acceleration

$\phi=$ angle

$\Omega=$ angular velocity

$\mu=$ dry friction coefficient

$\mu_{0}=$ static friction coefficient

$\mu_{k}=$ dynamic friction coefficient

$k_{i}=i$ th stiffness coefficient

$c_{i}=i$ th damping coefficient

$J=$ moment of inertia

$m_{i}=i$ th mass

$r=$ radius

$S=$ displacement after slip phase 


$$
\begin{aligned}
A & =\text { amplitude of vibrations } \\
t & =\text { time }
\end{aligned}
$$

\section{References}

[1] Ibrahim, R. A., 1994, "Friction-Induced Vibration, Chatter, Squeal, and Chaos, Part II: Dynamics and Modelling," Appl. Mech. Rev. 47(7), pp. 227-253.

[2] Jansen, J., 1993, "Nonlinear Dynamics of Oilwell Drill-Strings," Ph.D. Thesis, Delft University Press, Delft, The Netherlands.

[3] Van Den Steen, L. 1997, "Suppressing Stick-Slip-Induced Drillstring Vibrations: A Hyperstability Approach," Ph.D. Thesis, University of Twente, Enschede, The Netherlands.

[4] Moore, D. F., 1975, Principles and Applications of Tribology, Pergamon Press, Oxford.

[5] Coulomb, C. A., 1809, Theorie des Machines Simples-en Ayant en Regard au Frottement de Leures Parties et a la Roideur des Cordages, Bachelier, Paris.

[6] Bowden, F. P. and Tabor, D., 1954, Friction and Lubrication of Solids, Clarendon Press, Oxford.

[7] Kragielskiy, I. V., 1944, On the Influence of Pressure and Surfaces Dimension on Amount of a Friction Force, Mashinostroyemye, Moscow (in Russian)

[8] Kragielskiy, I. V. and Alisin, V. V., 1978, Friction, Wear and Lubrication, Mashinostroyemye, Moscow (in Russian).

[9] Kostecki, I. V., 1970, Friction, Wear and Lubrication in Machines, Tekhnika, Kiyew (in Russian).

[10] Epifanov, G. I., 1957, "On the Two-parts Friction," AN SSSR, Moscow (in Russian).

[11] Awrejcewicz, J. and Pyryev, Yu., 2002, "Thermoelastic Contact of a Rotating Shaft With a Rigid Bush in Conditions of Bush and Stick-Slip Movements," Int. J. Eng. Sci., 40, pp. 1113-1130.

[12] Mistakidis, E. S., Panagouli, O. K., and Panagiotopoulos, P. D., 1998, "Unilateral Contact Problems With Fractal Geometry and Fractal Friction Laws. Methods of Calculation," Comput. Mech., 21, pp. 353-362.

[13] Panagiotopoulos, P. D., Panagouli, O. K., and Mistakidis, E. S., 1994, "On the Consideration of the Geometric and Physical Fractality in Solid Mechanics, Part I: Theoretical Results," Z. Angew. Math. Mech. 74, pp. 167-176.

[14] Feeny, B., Guran, A., Hinrichs, N., and Popp, K., 1998, "A Historical Review on Dry Friction and Stick-Slip Phenomena," Appl. Mech. Rev. 51, pp. 321341.

[15] Ibrahim, R. A., 1994, "Friction-Induced Vibration, Chatter, Squeal, and Chaos, Part I: Mechanics of Contact and Friction," Appl. Mech. Rev., 47(7), pp. 209-226.

[16] Popp, K., Hinrichs, N., and Oestreich, M., 1996, Analysis of a Self-Excited Friction Oscillator With External Excitation, World Scientific, London.

[17] Andreaus, V. and Casini, P., 2000, "Dynamics of Friction Oscillators Excited by a Moving Base and/or Driving Force," J. Sound Vib., pp. 245(4), 685-699.

[18] Galvanetto, U., 2001, "Some Discontinuous Bifurcations in a Two-Block Stick-Slip System," J. Sound Vib., 248(4), pp. 653-669.

[19] Galvanetto, U., 2002, "Some Remarks on the Two-Block Symmetric BurridgeKnopoff Model,” Phys. Lett. A, 293, pp. 251-259.

[20] Galvanetto, U. and Bishop, S. R., 1995, "Characterisation of the Dynamics of a Four-Dimensional Stick-Slip System by a Scalar Variable," Chaos, Solitons Fractals, 5(11), pp. 2171-2179.

[21] Van De Vrande, B. L., Van Campen, D. H., and De Kraker, A., 1999, "An Approximate Analysis of Dry-Friction-Induced Stick-Slip Vibrations by a Smoothing Procedure," Nonlinear Dyn. 19, pp. 159-167.

[22] Awrejcewicz, J. and Olejnik, P., 2003, "Regular and Chaotic Stick-Slip Dynamics in a Self-Excited Two-Degrees-of Freedom System With Friction," Int. J. Bifurcation Chaos Appl. Sci. Eng., 4(13), pp. 843-861.

[23] Fu, W. P., Fang, Z. D., and Zhao, Z. G., 2001, "Periodic Solutions and Harmonic Analysis of an Anti-Lock Brake System With Piecewise-Nonlinearity," J. Sound Vib., 246(3), pp. 543-550.

[24] Choi, H. S. and Lou, J. Y. K., 1991, "Non-linear Behavior and Chaotic Motions of an SDOF System With Piecewise-Non-linear Stiffness," Int. J. NonLinear Mech., 26, pp. 461-473.

[25] Fling, R. T. and Fenton, R. E., 1981, "A Describing-Function Approach to Antiskid Design," IEEE Trans. Veh. Technol., 30(3), pp. 134-144.

[26] Perko, L., 1991, Differential Equations and Dynamical Systems, SpringerVerlag, New York.

[27] Yeh, E. C. and Day, G. C., 1992, "Parametric Study of Antiskid Brake Systems Using Poincaré Map Concept,” Int. J. Veh. Des., 13, pp. 210-232.

[28] Brandl, M. and Pfeiffer, F., 1999, "Tribometer for Dry Friction Measurement,' Trans. ASME, Design Eng. Technical Conf. and Computers and Information in Eng. Conf., Las Vegas, DETC99/VIB-8353.

[29] Dweib, A. H. and De Souza, A. F. 1990, "Self-Excited Vibrations Induced by Dry Friction, Part I: Experimental Study," J. Sound Vib., 137(2), pp. 163-175.

[30] Hartung, A., Schmieg, H., and Vlelsack, P., 2001, "Passive Vibration Absorber With Dry Friction," Arch. Appl. Mech., 71(6-7), pp. 463-472.

[31] Schmieg, H. and Vlelsack, P., 1998, "Modellbildung und experimentelle Untersuchungen zum Bremsenquietschen," Z. Angew. Math. Mech., 78, pp. 709710

[32] Tariku, F. A. and Rogers, R. J., 2001, "Improved Dynamic Friction Models for Simulation of One-dimensional and Two-dimensional Stick-Slip Motion," J. Tribol. 123, pp. 661-669.

[33] Karnopp, D. 1985, "Computer Simulation of Stick-Slip Friction in Mechanical Dynamic Systems," ASME J. Dyn. Syst., Meas., Control, 107, pp. 100-103.

[34] Tan, X. and Rogers, R. J. 1996, "Dynamic Friction Modelling in Heat Ex- changer Tube Simulations," Trans. ASME, Flow-Induced Vibrations, Vol. 328 pp. 347-358.

[35] Bogacz, R., and Ryczek, B. 1997, "Dry Friction Self-Excited Vibrations; Analysis and Experiment," Eng. Trans., 45(3-4), pp. 487-504.

[36] Bogacz, R., Irretier, H., and Sikora, J. 1990, "On Discrete Modelling of Contact Problems With Friction," Z. Angew. Math. Mech., 70(4), pp. T31-T32.

[37] Awrejcewicz, J. 1983, "Analysis of Self-Excited Vibrations Due to Nonlinear Friction in One Degree of Freedom System," Sci. Bull. Łódź Technical University, 66, pp. 21-28 (in Polish).

[38] Awrejcewicz, J. 1987, "Analysis of Self-Excited Vibration in Mechanical System With Four Degrees of Freedom,” Sci. Bull. Łódź Technical University, 72 , pp. 5-27.

[39] Bothe, D. 1999, "Periodic Solutions of Non-Smooth Friction Oscillators," Z. Angew. Math. Phys., 50, pp. 779-808.

[40] Fečkan, M., 1997, "Bifurcation of Periodic Solutions in Defferential Inclusions," Appl. Math. (Germany), 42(5), pp. 369-393.

[41] Fečkan, M., 1999, "Chaotic Solutions in Differential Inclusions: Chaos in Dry Friction Problems," Trans. Am. Math. Soc., 351(7), pp. 2861-2873.

[42] Filippov, A. F. 1988, Differential Equations With Discontinuous Right-Hand Sides, Kluwer, Dortrecht-Boston-London.

[43] Guckenheimer, J. and Holmes, P. 1983, Nonlinear Vibrations, Dynamical Systems and Bifurcation of Vector Fields, Springer-Verlag, New York.

[44] Kunze, M. 2000, Non-smooth Dynamical Systems, Springer-Verlag, BerlinHeidelberg.

[45] Kunze, M. and Küpper, T., 1997, "Qualitative Bifurcation Analysis of a NonSmooth Friction-Oscillator Model," Z. Angew. Math. Phys., 48, pp. 87-101.

[46] Dubois, A., Oudin, J., and Picart, P. 1996, "Elastoplastic Finite Element Analysis of an Upsitting-Sliding Test for the Determination of Friction at Medium and High Contact Pressure," Tribol. Int., 29(7), pp. 603-613.

[47] Guerin, J. D., Bartys, H., Dubois, A., and Oudin, J. 1999, "Finite Element Implementation of a Generalized Friction Model: Application to an UpsettingSliding Test”, Finite Elem. Anal. Design, 31, pp 193-207.

[48] Bay, N. 1987, "Friction Stress and Normal Stress in Bulk Forming Processes," J. Mech. Work. Technol., 14, 203-223.

[49] Wanheim, T. and Bay, N. 1978, "A Model for Friction in Metal Forming Processes," CIRP Ann., 27, pp. 189-194.

[50] Gaul, L. and Nitsche, R., 2001, "The Role of Friction in Mechanical Joints," Appl. Mech. Rev. 54(2), pp. 93-106.

[51] Ottl, D. 1981, Schwingungen mechanischer Systeme mit Strukturdämpfung, VDI-Fortschritt-Berichte 603, Düsseldorf.

[52] Stribeck, R. 1902, "Die Wesentlichen Eigenschaften der Gleit- und Rollenlager - The Key Qualities of Sliding and Roller Bearings," Zeitschrift des. Vereins deutscher Ingenieure 46(38-39), pp. 1342-1348, 1432-1437.

[53] Canudas de Wit, C., Olson, H., Àström, K. J., and Lischinsky, P. 1995, “ New Model for Control Systems With Friction," IEEE Trans. Autom. Control 40(3), pp. 419-425.

[54] Willner, K. and Gaul, L. 1995, "A Penalty Approach for Contact Description by FEM Based on Interface Physics," Proc. Contact Mechanics II, Ferrara, Italy, pp. 257-264.

[55] Majundar, A. and Bhushan, B. 1991, "Fractal Model of Elastic-Plastic Contac Between Rough Surfaces," J. Tribol. 113, pp. 1-11.

[56] Johnson, K. L. 1985, Contact Mechanics, Cambridge Univ. Press, Cambridge.

[57] Den Hartog, J. P, 1931, "Forced Vibrations With Combined Coulomb and Viscous Friction," ASME Paper No. APM-53-9, pp. 107-115.

[58] Levitan, E. S. 1960, "Forced Vibrations of a Spring-Mass System Having Combined Coulomb and Viscous Damping," J. Acoust. Soc. Am. 32(10), pp. 1265-1269.

[59] Yeh, G. C. K. 1966, "Forced Vibrations of a Two-Degree-of-Freedom System With Combined Coulomb and Viscous Damping," J. Acoust. Soc. Am., 39, pp. 14-24.

[60] Moreau, J. J. 1988, "Unilateral Contact Dry Friction in Finite Freedom Dynamics," Non-Smooth Mech. \& Applications, CISM Courses and Lectures, Springer Verlag, Wien.

[61] Pfeiffer, F. 1999, "Unilateral Problems of Dynamics," Arch. Appl. Mech., 69, 503-527.

[62] Wösle, M. and Pfeiffer, F. 1999, "Dynamics of Spatial Structure-Varying Rigid Multibody Systems," Arch. Appl. Mech. 69, pp. 265-285.

[63] Zwörner, O., Hölscher, H., Schwarz, U. D., and Wiesendanger, R. 1998, "The Velocity Dependence of Frictional Forces in Point-Contact Friction," Appl. Phys. A, 66, pp. S263-S267.

[64] Åström, K. J. and Wittenmark, B. 1995, Adaptive Control. Addison-Wesley, Massachusetts.

[65] Newton, G., Gould, L., and Kaiser, J. 1957, Analytical Design of Linear Feedback Controls, Wiley, New York.

[66] Walrath, C. 1984, "Adaptive Bearing Friction Compensation Based on Recent Knowledge of Dynamic Friction," Automatica, 20(6), pp. 717-727.

[67] Dahl, P. R. 1975, "Solid Friction Damping of Spacecraft Vibrations," AIAA Guidance and Control Conf, Boston, 75-1104.

[68] Dahl, P. R. 1976, "Solid Friction Damping of Mechanical Vibrations," AIAA J. 14(12), pp. 1675-1682.

[69] Ramberg, W. and Osgood, W. R. 1943, "Description of Stress-Strain Curves by Three Parameters," Tech. Note, National Advisory Committee for Aeronautics 902.

[70] Sargin, M. 1971, "Stress-Strain Relationship for Concrete and the Analysis of Structural Concrete Sections, SM Study, Solid Mech. Division, University of Waterloo 4.

[71] Åström, K. J., 1995, Control of Systems With Friction, Swedish Research 
Council for Eng. Science, 759.

[72] Bliman, P.-A., 1992, "Mathematical Study of the Dahl's Friction Model," Eur. J. Mech. A/Solids, 11(6), pp. 835-848.

[73] Krasnoselskiy, M. A. and Pokrovskiy, A. V. 1980, Systems With Hysteresis, Springer, New York.

[74] Bliman, P.-A. and Sorine, M. 1993, "A System Theoretic Approach of Systems With Hysteresis. Application to Friction Modelling and Compensation," Proc. of 2nd European Control Conf., Groningen, The Netherlands, pp. 1844-1849.

[75] Bliman, P.-A. and Sorine, M. 1995, "Easy-to-Use Realistic Dry Friction Models for Automatic Control," Proc. of 3rd European Control Conf. Rome, Italy, pp. 3788-3794.

[76] Rabinowicz, E. 1951, "The Nature of Static and Kinetic Coefficients of Friction,” J. Appl. Phys., 22(11), pp. 1373-1379.

[77] Altpeter, F., Ghorbel, F., and Longchamp, R. 1998, "Relationship Between Two Friction Models: A Singular Perturbation Approach," Proc. 37th IEEE Conf. on Decision and Control, Florida, USA, pp. 1572-1574.

[78] Choi, H. S. and Lou, J. Y. K. 1991, "Non-linear Behavior and Chaotic Motions of an SDOF System With Piecewise-Non-linear Stiffness," Int. J. Non-Linear Mech., 26, pp. 461-473.

[79] Ostermeyer, G. P. 2001, "Friction and Wear of Brake Systems," Forschung im Ingenieurwesen, 66(6), pp. 267-272

[80] Eriksson, M. and Jacobson, S. 2000, "Tribological Surfaces of Organic Brake Pads," Tribol. Int., 33, pp. 817-827.

[81] Stoker, J. J. 1950, Nonlinear Vibrations, Wiley, New York.

[82] Hassard, B. D., Kazarinoff, N. D., and Wan, Y. H. 1981, Theory and Applications of Hopf Bifurcation, Cambridge Univ. Press, Cambridge.

[83] Awrejcewicz, J. 1986, "Chaos in Simple Mechanical Systems With Friction," J. Sound Vib., 109(1), pp. 178-180.

[84] Awrejcewicz, J. and Krodkiewski, J. 1984, "Analysis of Self-Excited Vibrations Due Nonlinear Friction in Two Degrees of Freedom System," Sci. Bull. Łódź Technical University, 67, pp. 13-30, (in Polish).

[85] Feely, O. and Chua, L. O. 1992, "Nonlinear Dynamics of a Class of Analogto-Digital Converters," Int. J. Bifurcation Chaos Appl. Sci. Eng. 2(2), pp. 325-340.

[86] Littlehales, P. A., Lewis, C. P., and Bishop, S. R., 1993, "Basins and Chaos in Sigma Delta Modulators," Proc. 9th Int. Conf. on Systems Engineering, Las Vegas, pp. 597-601.

[87] Burridge, R. and Knopoff, L., 1967, "Model and Theoretical Seismicity," Bull. Seismol. Soc. Am., 57(3), pp. 341-371.

[88] Cao, T. and Aki, K., 1986, "Seismicity Simulation With Rate- and StateDependent Friction Law," PAGEOPH, 124(3), pp. 487-513.

[89] Carlson, J. M. and Langer, J. S., 1989, "Properties of Earthquakes Generated by Fault Dynamics,” Phys. Rev. Lett., 62(22), pp. 2632-2635.

[90] Nussbaum, J. and Ruina, A., 1987, "A Two Degree-of-Freedom Earthquake Model With Static/Dynamic Friction," PAGEOPH 125(4), pp. 629-656.

[91] Knudsen, C., Feldberg, R., and Jaschinski, A., 1991, "Non-linear Dynamic Phenomena in the Behaviour of a Railway Wheelset Model," Nonlinear Dyn., 2, pp. 389-404.

[92] Hinrichs, N., Oestreich, M., and Popp, K., 1997, "Dynamics of Oscillators With Impact and Friction," Chaos, Solitons Fractals, 8(4), pp. 535-558.

[93] Merchant, M. E., 1944, "Basic Mechanics of the Metal Cutting Process," ASME J. Appl. Mech., 66, pp. 168-175.

[94] Minis, I. E., Magrab, E. B., and Pandelidis, I. O., 1990, "Improved Methods for the Prediction of Chatter in Turning, Part 2: Determination of Cutting Process Parameters," ASME J. Eng. Ind., 112, pp. 21-32.

[95] Tobias, S. A. (1965) Machine Tool Vibration, Wiley, New York.

[96] Tłusty, J., 1978, "Analysis of the State of Research in Cutting Dynamics," CIRP Ann., 27, pp. 583-595.

[97] Stefański, A., Wojewoda, J., and Furmanik, K., 2000, "Experimental and Numerical Analysis of Self-Excited Friction Oscillator," Chaos, Solitons Fractals, 12, pp. 1691-1704.

[98] Cunningham, W. J., 1958, Introduction to Nonlinear Analysis, McGraw Hill, New York.

[99] Hayashi, Ch., 1964, Non-linear Vibrations in Physical Systems, McGraw Hill, New York.

[100] Minorsky, N., 1962, Nonlinear Vibrations in Physical Systems, McGraw Hill, New York.

[101] Awrejcewicz, J., 1990, "Parametric and Self-Excited Vibrations Induced by Friction in a System With Three Degrees of Freedom,” KSME Int. J., 4(2), pp. $156-166$

[102] Brommundt, E., 1974, "On the Numerical Investigation of Nonlinear Periodic Rotor Vibrations," Dynamics of Rotors IUTAM, Lyngby, pp. 75-102.

[103] Naranayanan, S. and Jayaraman, K., 1989, "Chaotic Motion in Nonlinea System With Coulomb Damping," Nonlinear Dynamics in Eng. Systems, IUTAM Symp., Stuttgart, pp. 217-224.

[104] Urabe, M., 1967, Nonlinear Autonomous Vibrations, Academic Press, New York

[105] Deimling, K., 1992, Multivalued Differential Equations, de Gruyter, Berlin New York.

[106] Den Hartog, J. P., 1930, "Forced Vibrations With Combined Viscous and Coulomb Damping," Philos. Mag., VII(9), pp. 801-817.

[107] Reissig, R., 1954, "Erzwungene Schwingungen mit zäher und trockner Reibung," Math. Nachr., 11, pp. 345-384

[108] Szablewski, W., 1954, "Einfluss der Coulombschen Reibung auf Schwingungsvorgänge," Math. Nachr. 12, pp. 183-208.

[109] Hong, H.-K. and Liu, C.-S., 2001, "Non-Sticking Vibration Formulae for Coulomb Friction Under Harmonic Loading," J. Sound Vib., 244(5), pp.
883-898

110] Chen, L. Y., Chen, J. T., Chen, C. H., and Hong, H.-K., 1994, "Free Vibration of a SDOF System With Hysteretic Damping," Mech. Res. Commun., 21, pp. 599-604

[111] Hundal, M. S., 1979, "Response of a Base Excited System With Coulomb and Viscous Friction," J. Sound Vib., 64, pp. 371-378.

[112] Beucke, K. E. and Kelly, J. M., 1985, "Equivalent Linearizations for Practical Hysteretic Systems," Int. J. Non-Linear Mech., 23, pp. 211-238.

[113] Nayfeh, A. H. and Mook, D. T., 1979, Nonlinear Vibrations, Wiley, New York.

[114] Awrejcewicz, J., 1996, Vibrations of Discrete Deterministic Systems, WNT, Warsaw (in Polish)

[115] Hong, H.-K. and Liu, C.-S., 2000 "Coulomb Friction Oscillator: Modelling and Responses to Harmonic Loads and Base Excitations," J. Sound Vib. 229, pp. 1171-1192.

[116] Awrejcewicz, J. and Olejnik, P., 2002, "Numerical Analysis of Self-Excited by Friction Chaotic Vibrations in Two-Degrees-of-Freedom System Using Hénon Method," Machine Dynamics Problems, 26(4), pp. 9-20.

[117] Hénon, M., 1982, "On the Numerical Computation of Poincaré Maps," Physica D, 5, pp. 412-413.

[118] Awrejcewicz, J. and Olejnik, P., 2000, "Regular and Chaotic Dynamics of the Two Degrees-of-Freedom System," XIX Symposium - Vih in Physical Systems, Poznań - Błażejewko, pp. 51-52.

[119] Awrejcewicz, J. and Olejnik, P., 2001, "Stick-Slip Dynamics of a TwoDegrees-of Freedom System," Trans. ASME, Design Eng. Technical Conf. and Computers and Information in Eng. Conf., Pittsburgh, DETC01/VIB21611

[120] Carlson, J. M. and Langer, J. S., 1989 "Mechanical Model of an Earthquake Fault," Phys. Rev. A, 40(11), pp. 6470-6484.

[121] Carlson, J. M., Langer, J. S., Shaw, B. E., and Tang, C., 1991, "IntrinsicProperties of a Burridge Knopoff Model of an Earthquake Fault," Phys. Rev. A, 44(2), pp. 884-897.

[122] Persson, B. N., 1998, "Sliding Friction: Physical Principles and Applications," Springer, Berlin.

[123] Oden, J. T., 1985, "Models and Computational Methods For Dynamic Friction Phenomena," Comput. Methods Appl. Mech. Eng., 52, pp. 527-634.

[124] Brommundt, E., 1995, "Ein Reibschwinger mit Selbsterregung ohne fallende Reibkennlinie,” Z. Angew. Math. Mech., 75, pp. 811-820.

[125] Wikiel, B. and Hill, J. M., 2000, "Stick-Slip Motion for Two Coupled Masses With Side Friction,” Int. J. Non-Linear Mech., 35, pp. 953-962.

[126] Guran, A., Pfeiffer, F., and Popp, K., 1996, Stability, Vibration and Control of Systems, Chapter 1, World Scientific

[127] Tołstoj, D. M., 1967, "Significance of the Normal Degree of Freedom and Natural Vibrations in Contact Friction," Wear, 10, pp. 199-213.

[128] Iwan, W. D. and Moeller, T. L., 1976, "The Stability of a Spinning Disc With a Transverse Load System,” ASME J. Appl. Mech., 43, pp. 485-490.

[129] Iwan, W. D. and Stahl, K. J., 1973, "The Response of an Elastic Disc With a Moving Mass System," ASME J. Appl. Mech., 40, pp. 445-451.

[130] Mote, C. D., 1970, "Stability of Circular Plate Subjected to Moving Loads," J. Franklin Inst., 290, pp. 329-344.

[131] Mote, C. D., 1977, "Moving Load Stability of a Circular Plate on a Floating Central Collar," J. Acoust. Soc. Am., 61, pp. 439-447.

[132] Ono, K., Chen, J.-S., and Bogy, D. B., 1991, "Stability Analysis for the Head-Disc Interface in a Flexible Disc Drive,” ASME J. Appl. Mech., 58, pp. 1005-1014.

[133] Miwa, T., 1967, "Evaluation Methods for Vibration Effect," Ind. Health, 5, pp. 183-205.

[134] Sissala, M., Heimola, T., and Otala, M., 1985, "Optimization of Lift Car Vibrational Behaviour by Modal Analysis," Elevator World, 6, pp. 27-31.

[135] Wee, H., Kim, Y. Y., Jung, H., and Lee, G. N., 2001, "Nonlinear RateDependent Stick-Slip Phenomena: Modeling and Parameter Estimation," Int J. Solids Struct., 38, pp. 1415-1431.

[136] Bowden, F. P. and Tabor, D., 1950, The Friction and Lubrication of Solids, Clarendon Press, Oxford.

137] Deryaguin, B. V., Push, V. E., and Tołstoj, D. M., 1957, "A Theory of StickSlip of Sliding Solids," Proc. Conf. Lubr. and Wear, London, pp. 255-268.

[138] Halling, J., 1975, Principles of Tribology, Macmillan Press, London.

[139] Kato, S., Yamaguchi, K., and Matsubayashi, T., 1974, "Stick-Slip Motion of Machine Tool Slideway," ASME J. Eng. Ind., 96, pp. 557-566.

[140] Singh, B. R. (1960) "Study of Critical Velocity of Stick-Slip Sliding," ASME J. Eng. Ind., 82, pp. 393-398.

[141] Van De Velde, F., and De Baets, P., 1997, "Comparison of Two Stick-Slip Testers and Recommendations for Repeatable and Significant Stick-Slip Testing," Tribotest, 3(3-4), pp. 361-392.

[142] Armstrong-Hélouvry, B., 1990, "Stick-Slip Arising From Stribeck Friction," Proc. IEEE, Int.Conf. Robotics and Automation, pp. 1377-1383.

[143] Banerjee, K., 1968, "Influence of Kinetic Friction on the Critical Velocity of Stick-Slip Motion," Wear, 12, pp. 107-116.

[144] Bell, R. and Burdekin, M., 1970, "A Study of Stick-Slip Motion of Machine Tool Feed Drives," Proc. Inst. Mech. Eng., 184, pp. 543-557.

[145] Bo, L. C. and Pavelescu, D., 1982, "The Friction-Speed Relation and Its Influence on the Critical Velocity of Stick-Slip Motion," Wear, 82, pp. 277 289.

[146] Brockley, C. A., Cameron, R., and Potter, A. F., 1967, "Friction-Induced Vibration," ASME J. Lubr. Technol., 89, pp. 101-108.

[147] Capone, G., D’Agostino, V., Della Valle, S., and Guida, D., 1993, "Influence of the Variation Between Static and Kinetic Friction on Stick-Slip Instabil- 
ity," Wear, 161, pp. 121-126.

[148] Cockerham, G. and Cole, M., 1976, "Stick-Slip Stability by Analogue Simulation," Wear, 36, pp. 189-198.

[149] Cockerham, G. and Symmons, G. R., 1976, "Stability Criterion for Stick-Slip Motion Using a Discontinuous Dynamic Friction Model," Wear, 40, pp. 113-120.

[150] Hunt, J. B., Torbe, I., and Spencer, G. C., 1965, "The Phase-Plane Analysis of Sliding Motion," Wear, 8, pp. 455-465.

[151] Van De Velde, F., and De Baets, P., 1998, "A New Approach of Stick-Slip Based on Quasi-Harmonic Tangential Vibrations," Wear, 216, pp. 12-26.

[152] You, H. I. and Hsia, J. H., 1995, "The Influence of Friction-Speed Relation on the Occurrence of Stick-Slip Motion," ASME J. Tribol., 117, pp. 450455.

[153] Gao, C. and Kuhlmann-Wilsdorf, D., 1990, "On Stick-Slip and the Velocity Dependence of Friction at Low Speeds," ASME J. Tribol., 112, pp. 354-360.

[154] Goeleven, D., Motreanu, D., and Motreanu, V. V., 2003, "On the Stability of Stationary Solutions of First Order Parabolic Variational Inequalities," Advances in Nonlinear Variational Inequalities, 6, pp. 1-30.

[155] Lozano, R., Brogliato, B., Egeland, O., and Maschke, B., 2000, Dissipative Systems Analysis and Control, Springer CCES, London.

[156] Komanduri, R., 1993, "Machining and Grinding - A Historical Review of Classical Papers," Appl. Mech. Rev., 46(3), pp. 80-132.

[157] Ramachandran, R., Pande, S. S., and Ramakrishnan, N., 1994, "The Role of Deburring in Manufacturing: A State-of-the-Art Survey," J. Mechanicals Processing Technol., 44, 1-13.

[158] Shia, C.-Y., Stango, R. J., and Heinrich, S. M., 1998, "Analysis of Contact Mechanics for a Circular Filamentary Brush/Workpart System," ASME J. Manuf. Sci. Eng., 120(4), pp. 715-721.

[159] Studny, D., Rittel, D., and Zussman, E., 1999, "Impact Fracture of Screws for Disassembly,” ASME J. Manuf. Sci. Eng., 121(1), pp. 118-126.

[160] Brogliato, B., Niculescu, S. I., and Orhant, P., 1997, "On the Control of Finite Dimensional Mechanical Systems With Unilateral Constraints," IEEE Trans. Autom. Control, 42(2), pp. 200-215.

[161] Brogliato, B., Niculescu, S. I., and Monteiro-Marques, M. D. P., 2000, "On Tracking Control of a Class of Complementary-Slackness Mechanical Systems," Syst. Control Lett., 39(4), pp. 255-266.

[162] Bourgeot, J.-M. and Brogliato, B. [YEAR], "Tracking Control of Complementarity Lagrangian Systems," Int. J. Bif. \&amp;amp;amp; Chaos (to appear).

[163] Van De Velde, F., and De Baets, P., 1996, "Mathematical Approach of the Influencing Coefficients on Stick-Slip Induced by Decelerative Motion," Wear, 201, pp. 80-93

[164] De Baets, P., Degrieck, J., Van De Velde, F., and Van Peteghem, A. P., 2000, "Experimental Verification of the Mechanisms Causing Stick-Slip Motion Originating From Relative Deceleration," Wear, 243, pp. 48-59.

[165] Brace, W. F. and Byerlee, J. D., 1996, "Stick-Slip as a Mechanism for Earthquakes," Science, 153, pp. 990-992.

[166] Bowden, F. P. and Tabor, D., 1939, "The Area of Contact Between Stationary and Between Moving Surfaces,” Proc. R. Soc. London, 169, pp. 391-413.

[167] Brune, J. N., Brown, S., and Johnson, P. A., 1993, "Rupture Mechanism and Interface Separation in Foam Rubber Models of Earthquakes: A Possible Solution to the Heat Flow Paradox and the Paradox of Large Overthrusts," Tectonophysics, 218, pp. 59-67.

[168] Brune, J. N., Johnson, P. A., and Slater, C., 1990, "Nucleation Predictability, and Rupture Mechanism in Foam Rubber Models of Earthquakes," J. Himalayan Geol., 1, 155-166.

[169] Anooshehpoor, R. and Brune, J. N., 1994, "Frictional Heat Generation and Seismic Radiation in a Foam Rubber Model of Earthquakes," PAGEOPH, 142, pp. 735-747.

[170] Comninou, M. and Dundurs, J., 1977, "Elastic Interface Waves Involving Separation,” ASME J. Appl. Mech., 44, pp. 222-226.

[171] Comninou, M. and Dundurs, J. (1978) "Can Two Solids Slide Without Slipping?,” Int. J. Solids Struct., 14, pp. 251-260.

[172] Comninou, M. and Dundurs, J., 1978, "Elastic Interface Waves and Sliding Between Two Solids," ASME J. Appl. Mech., 45, pp. 325-330.

[173] Tworzydlo, W. W. and Hamzeh, O. N., 1997, "On the Importance of Normal Vibrations in Modelling of Stick-Slip In Rock Sliding," J. Geophys. Res., 102, pp. 15091-15103.

[174] Bouissou, S., Petit, J. P., and Barquins, M., 1998, "Experimental Evidence of Contact Loss During Stick-Slip. Possible Implications for Seismic Behaviour," Tectonophysics, 295, pp. 341-350.

[175] Bouissou, S., Petit, J. P., and Barquins, M., 1998, "Normal Load, Slip Rate and Roughness Influence on the PMMA Dynamics of Sliding, Part 1: Stable Sliding to Stick-Slip Transition," Wear, 214, pp. 156-164.

[176] Bouissou, S., Petit, J. P., and Barquins, M., 1998, "Normal Load, Slip Rate and Roughness Influence on the PMMA Dynamics of Sliding, Part 2: Characterisation of the Stick-Slip Phenomenon," Wear, 215, pp. 137-145.

[177] Antoniou, S. S., Cameron, A., and Gentle, C. R., 1976, "The Friction-Speed Relation From Stick-Slip Data," Wear, 36, pp. 235-254

[178] Bell, R. and Burdekin, M., 1969, "A Study of the Stick-Slip Motion of Machine Tool Feed Drives," Proc. Inst. Mech. Eng., 184(29), pp. 543-560.

[179] Cockerham, G. and Symmons, G. R., 1978, "Stick-Slip Stability by Transfer Lubrication," Proc. Inst. Mech. Eng., 192, pp. 259-268.

[180] Hess, D. P. and Soom, A., 1990, "Friction at a Lubricated Line Contactoperating at Oscillating Sliding Velocities," ASME J. Tribol., 112, pp. 147-152.

[181] Lee, R. T., Yang, C. R., and Chiou, Y. C., 1996, "A Procedure for Evaluating the Positioning Accuracy of Reciprocating Friction Drive Systems," Tribol.
Int., 29(5), pp. 394-404.

182] Marui, E. and Endo, H., 1996, "Some Considerations of Slideway Friction Characteristics by Observing Stick-Slip Vibration,” Tribol. Int., 29(3), pp. 251-262.

[183] Sakamoto, T., 1985, "Normal Displacement of the Sliding Body in a StickSlip Friction Process," Proc. JSLE, Int. Trib. Conf., Tokyo, pp. 141-146.

[184] Sakamoto, T., 1987, "Normal Displacement and Dynamic Friction Characteristics in a Stick-Slip Process," Tribol. Int., 20(1), pp. 25-31.

[185] Sampson, J. B., Morgan, F., Reed, D. W., and Muskat, M., 1943, "Friction Behaviour During the Slip Portion of the Stick-Slip Process," J. Appl. Phys., 14, pp. 689-700.

[186] Van De Velde, F. and De Baets, P., 1998, "The Relation Between Friction Force and Relative Speed During the Slip-Phase of a Stick-Slip Cycle," Wear, 219, pp. 220-226.

187] Tabor, D., 1981, "Friction," ASME J. Lubr. Technol. 103, pp. 169-179.

[188] Zhuravlev, V. G., 1998, "The Model of Dry Friction in the Problem of the Rolling of Rigid Bodies,” J. Appl. Math. Mech., 62(5), pp. 705-710.

[189] Brockley, C. A. and Davis, H. R., 1968, "The Time Dependence of Static Friction," ASME J. Lubr. Technol., 90, pp. 35-41.

[190] Dietrich, J. H., 1972, "Time-Dependent Friction in Rocks," J. Geophys. Res. 77, pp. 110-122.

[191] Pavelescu, D. and Tudor, A., 1987, "The Sliding Coefficient: Its Evolution and Usefulness," Wear, 120, pp. 321-336.

[192] Rabinowicz, E., 1958, "The Intrinsic Variables Affecting the Stick-Slip Process," Proc. Phys. Soc. London 71, pp. 668-675.

[193] Ferrero, J. F. and Barrau, J. J., 1996, "Study of Dry Friction Under Small Displacements and Near-Zero Sliding Velocity," Wear, 209, pp. 322-327.

[194] Tondl, A. and Nabergoy, R., 1994, "Non-Periodic and Chaotic Vibrations in a Flow Induced System," Chaos, Solitons Fractals, 4(3), pp. 2193-2202.

[195] Tondl, A. and Nabergoy, R., 1995 "A Flow Induced System With Friction," Chaos, Solitons Fractals, 7(3), pp. 353-363.

[196] Aubin, J. P. and Celina, A., 1984, Differential Inclusions, Springer Verlag, Berlin-Heidelberg-New York.

[197] Deimling, K., Hetzer, G., and Shen, W. X., 1996, "Almost Periodicity En forced by Coulomb Friction,” Adv. Differ. Equ., 1, pp. 265-281.

[198] Eckmann, J. P. and Ruelle, D., 1985, "Ergodic Theory of Chaos and Strange Attractors," Rev. Mod. Phys., 57, pp. 617-656.

[199] Georgiadis, F., Vakakis, A. F., McFarland, D. M., and Bergman, L., "Shock Isolation Through Passive Energy Pumping Caused by Non-Smooth Nonlinearities," Int. J. Bifurcation Chaos Appl. Sci. Eng. (to appear).

[200] Fečkan, M., "Chaos in Non-Autonomous Differential Inclusions," Int. J. Bifurcation Chaos Appl. Sci. Eng. (to appear).

[201] Matrossov, I. V., 2001, "On Existence and Uniqueness of Solutions for Equations of Motion of Mechanical System With Dry Friction," Nonlinear Anal. Theory, Methods Appl., 47, pp. 5391-5402.

[202] Matrossov, V. M. and Finogenko, I. A., 1996, "On Existence of the Right Side Solutions of the Differential Equations of Mechanical Systems With Dry Friction," Diff. Eq., 32(5), pp. 606-614.

[203] Feeny, B. and Moon, F. C., 1994, "Chaos in a Forced Dry Friction Oscillator: Experiment and Numerical Modelling," J. Sound Vib., 170(3), pp. 303-323.

[204] Shaw, S. W., 1986, "On the Dynamic Response of a System With Dry Friction," J. Sound Vib., 108, pp. 305-325.

[205] Wojewoda, J., Kapitaniak, T., Barron, R., and Brindley, J., 1993, "Complex Behaviour of a Quasiperiodically Forced System With Friction," Chaos, Solitons Fractals, 3(1), pp. 35-46.

[206] Lorenz, E. N., 2002, "Deterministic Nonperiodic Flow," J. Atmos. Sci., 10, pp. $130-138$

[207] Ueda, Y., 1979, "Randomly Transitional Phenomena in the System Governed by Duffing Equation,” J. Stat. Phys., 20, pp. 181-186.

[208] Awrejcewicz, J., 1986, "On the Occurence of Chaos in Duffing's Oscillator," J. Sound Vib., 108(1), pp. 176-178.

[209] Szemplińska-Stupnicka, W., 1987, "Secondary Resonances and Approximate Models of Routes to Chaotic Motion in Nonlinear Vibrations," J. Sound Vib. 113(1), pp. 155-172

[210] Troger, H., 1982, "Chaotic Behaviour in a Simple Mechanical Systems," Z Angew. Math. Mech., 62, pp. 18-27.

[211] Ageno, A. and Sinopoli, A., Lyapunov's Exponents for Non-Smooth Dynamics With Impacts: Stability Analysis of the Rocking Block," Int. J. Bifurcation Chaos Appl. Sci. Eng. (to appear).

[212] Galvanetto, U., Bishop, S. R., and Briseghella, L., 1995, "Some Remarks on the Stick-Slip Vibrations of a Two-Degree-of-Freedom Mechanical Mode,' Mech. Res. Commun., 20(6), pp. 459-466.

[213] Awrejcewicz, J. and Holicke, M. M., 1999, "Melnikov's Method and StickSlip Chaotic Vibrations in Very Weakly Forced Mechanical Systems," Int. J. Bifurcation Chaos Appl. Sci. Eng., 9(3), pp. 505-518.

[214] Awrejcewicz, J., 1989 Bifurcation and Chaos in Simple Dynamical Systems. World Scientific, Singapore.

[215] Awrejcewicz, J., 1991, Bifurcation and Chaos in Coupled Oscillators, World Scientific, Singapore.

[216] Maistrenko, V., Maistrenko, Y., and Sushko, I., 1994, "Noninvertible TwoDimensional Maps Arising in Radiophysics," Int. J. Bifurcation Chaos Appl. Sci. Eng., 4(2), pp. 383-400.

[217] Mira, C. (1981), "Complex Dynamics in Two-Dimensional Endoporphisms," Nonlinear Anal. Theory, Methods Appl., 4, 1167-1187.

[218] Stewart, H. B. and Lansburg, A. N., 1992 "Forecasting Catastrophe by Exploring Chaotic Dynamics," Appl. Chaos, 2, pp. 393-410. 
[219] Szemplińska-Stupnicka, W., 1990, The Behavior of Nonlinear Vibrating System, Kluwer, Dordrecht.

[220] Oseledec, V. I., 1968, "A Multiplicative Ergodic Theorem: Lyapunov Characteristic Numbers for Dynamical Systems," Trans. Mosc. Math. Soc., 19, pp. 197-231.

[221] Benettin, G., Galgani, L., Giorgilli, A., and Strelcyn, J. M., 1980, "Lyapunov Exponents for Smooth Dynamical Systems and Hamiltonian Systems; A method for Computing all of Them, Part 1: Theory," Meccanica, 15, pp. 9-20.

[222] Benettin, G., Galgani, L., Giorgilli, A., and Strelcyn, J. M., 1980, "Lyapunov Exponents for Smooth Dynamical Systems and Hamiltonian Systems; A method for Computing All of Them, Part 2: Numerical Application," Meccanica, 15, pp. 21-30.

[223] Wolf, A., Swift, J. B., Swinney, H. L., and Vastano, J. A., 1985, "Determining Lyapunov Exponents From a Time Series,” Physica D, 16, pp. 285-317.

[224] Muller, P., 1995, "Calculation of Lyapunov Exponents for Dynamical Systems With Discontinuities," Chaos, Solitons Fractals, 5(9), pp. 1671-1681.

[225] Pecora, L. M. and Carroll, T. L., 1990, "Synchronization of Chaos," Phys. Rev. Lett., 64, pp. 221-224.

[226] Stefański, A., 2000, "Estimation of the Largest Lyapunov Exponent in Systems With Impacts," Chaos, Solitons Fractals, 11, pp. 2443-2451.

[227] Stefański, A. and Kapitaniak, T., 2000, "Using Chaos Synchronization to Estimate the Largest Lyapunov Exponent of Nonsmooth Systems," Discrete Dyn. Nat. Soc., 4, pp. 207-215.

[228] Fujisaka, H. and Yamada, T., 1983 "Stability Theory of Synchronized Motion in Coupled-Oscillator Systems," Prog. Theor. Phys., 69(1), pp. 32-47.

[229] Awrejcewicz, J. and Olejnik, P., 2002, "Calculating Lyapunov Exponents From an Interpolated Time Series," XX Symposium - Vib. in Physical Systems, Poznan - Błażejewko, pp. 94-95

[230] Renard, Y., 2001, "Numerical Analysis of a One-Dimensional Elastodynamic Model of Dry Friction and Uni-Lateral Contact," Comput. Methods Appl. Mech. Eng., 190(15-17), pp. 2031-2050.

[231] Sokolov, I. Y., 2002, "Anomalous Increase of Friction in the Vicinity of Nano-Size Defects," Tribol. Lett., 12(2), pp. 131-134.

[232] Vidall-Sallé, E., Baillet, L., and Boyer, J. C., 2001, "Friction Law for Hydrostatic Mixed Lubrication Regime," J. Mater. Process. Technol., 118(1-3), pp. 101-108.

[233] Ahn, S. W., 2001, "The Effects of Roughness Types on Friction Coefficients and Heat Transfer in Roughened Rectangular Duct," Int. Commun. Heat Mass Transfer, 28(7), pp. 933-942.

[234] Marx, D. T. and Policandriotes, T., 2001, "Measurement of Interfacial Temperatures During Testing of a Subscale Aircraft Brake," J. Phys. D, 34, pp. 976-984

[235] Ostermeyer, G. P., 1999, “A Mesoscopic Particle Method for Description of Thermo-Mechanical and Friction Processes," Physical Mesomechanics, 2(6), pp. 33-39.

[236] Fleury, E., 2002, "Articles - Sliding Friction and Wear Behavior of Al-NiCo-Si Quasi-Crystalline Coatings Deposited by the High-Velocity Oxy-Fuel Spraying Technique," J. Mater. Res., 17(2), pp. 492-501.

[237] Göken, J. and Riehemann, W., 2002, "Dependence of Internal Friction of
Fibre-Reinforced and Unreinforced AZ91 on Heat Treatment," Mater. Sci. Eng., A, 324(1-2), pp. 127-133.

[238] Trojanova, Z., 2002, "Study of Relaxation of Residual internal Stress in Mg Composites by Internal Friction," Mater. Sci. Eng., A, 324(1-2), pp. 122126.

[239] Kiselev, A. S., 2002, "Loss of Specific Impulse Due to Friction in Spike Nozzles," Fluid Dyn., 36(5), pp. 743-750.

[240] Ko, J. S. and Gellman, A. J., 2001, "Molecular Layering Effects on Friction at $\mathrm{Ni}(100) / \mathrm{Ni}(100)$ Interfaces," J. Phys. Chem. B, 105(22), pp. 5186-5195.

[241] Zhongqing, W., Chen, W., and Chunli, B., 2001, "Investigation of Nanoscale Frictional Contact by Friction Force Microscopy," Langmuir, 17(3), pp. 3945-3951.

[242] Grabec, I., 1986, "Chaos Generated by the Cutting Process," Phys. Lett. A, 117, pp. 236-384.

[243] Larsson, R., 2001, "Base Fluid Parameters for Elastohydro-Dynamic Lubrication and Friction Calculations and Their Influence on Lubrication Capability," J. Synthetic Lubrication, 18(3), pp. 183-198.

[244] Awrejcewicz, J. and Delfs, J., 1990, "Dynamics of a Self-Excited Stick-Slip Oscillator With Two Degrees of Freedom, Part 1: Investigation of Equilibria," Eur. J. Mech. A/Solids, 9(4), pp. 269-282.

[245] Awrejcewicz, J. and Delfs, J., 1990, "Dynamics of a Self-Excited Stick-Slip Oscillator With Two Degrees of Freedom, Part 2: Slip-Stick, Slip-slip, StickSlip Transitions, Periodic and Chaotic Orbits," Eur. J. Mech. A/Solids, 9(5), pp. 397-418.

[246] Monteiro-Marques, M. D. P., 1994, “An Existence, Uniqueness and Regularity Study of the Dynamics of Systems With One-Dimensional Friction," Eur J. Mech. A/Solids, 13(2), pp. 277-306.

[247] Popp, K. and Stelter, P., 1989, Nonlinear Vibrations of Structures Induced by Dry Friction, Springer-Verlag, Berlin.

[248] Pratt, T. K. and Williams, R., 1981, "Nonlinear Analysis of Stick-Slip Motion," J. Sound Vib., 74(4), pp. 531-542.

[249] Qiao, S. L. and Ibrahim, R. A., 1999, "Stochastic Dynamics of Systems With Friction-Induced Vibration,” J. Sound Vib., 223(1), pp. 115-140.

[250] Hulten, J. O., 1997, "Friction Phenomena Related to Drum Brake Squeal Instabilities," Proc. ASME Design Eng. Tech. Conf. 16th Biennial Conf. Mech. Vib. \& Noise, Sacramento.

[251] Ostermeyer, G. P., 2001, "Friction and Wear of Brake Systems," Forschung im Ingenieurwesen, 66(6), pp. 267-272.

[252] Awrejcewicz, J. and Olejnik, P., 2003, "Mathematical Modeling and Simulation of a Girling Duo-Servo Brake," Proc. 29th Int. Summer School AMEE, Sozopol, Bulgaria [to appear].

[253] Awrejcewicz, J. and Olejnik, P., 2003, "Numerical and Experimental Investigations of Simple Non-linear System Modelling a Girling Duo-Servo Brake Mechanism," Proc. of DETC2003, ASME 2003 Design Engineering Technical Conferences and Computers and Information in Engineering Conference. September 2-6, Chicago, Illinois, USA, CD ROM, 7 pages.

[254] Awrejcewicz, J. and Olejnik, P., 2003, "Occurrence of Stick-Slip Phenomenon in a Real Laboratory Rig," Proc. of the 7th Conference on Dynamical Systems - Theory and Applications, Awrejcewicz, J., Owczarek, A., and Mrozowski, J., (eds.) Łódź, Poland, December 8-11, pp. 131-138.

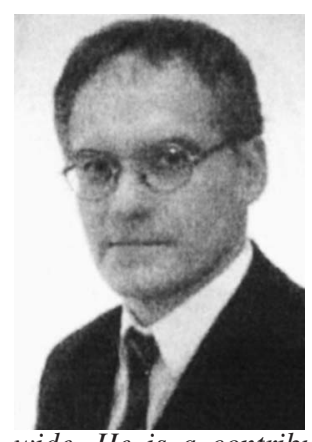

Jan Awrejcewicz was born in Telesze, Poland in 1952. He received the M.Sc. and Ph.D. degrees in the field of Mechanics from the Lodz. Technical University in 1977 and 1981, respectively. He also earned his bachelor's degree in Philosophy in 1978 from Lodz. University, and his D.Sc. degree (habilitation) in Mechanics from the Technical University of Lodz in 1990. In 1994, he earned the title of Professor from the President of Poland, Lech Walesa. In 1997, he became an Ordinary Professor (Ministry of Education of Poland). He is now the Chairperson of the Department of Automatics and Biomechanics, the Ph.D. School in Mechanics, and the Committee for Science of the Faculty of Mechanical Engineering of the Technical University of Lodz. He has authored or coauthored 27 monographs, 2 textbooks, 2 edited volumes, 9 conference proceedings, 5 journal special issues, 4 other books, 200 papers in scientific journals and chapters in books, 139 papers in the proceedings of international conferences, 50 papers in the proceedings of national conferences, and 124 short communications and technical reports. He has also delivered 40 lectures during the international conferences and gave 32 invited seminars at renowned universities worldwide. He is a contributor to 50 different journals and to 110 conferences. His papers and research cover various disciplines of mechanics, biomechanics, mathematics, physics and computer oriented sciences (for more details see www.p.lodz.pl/K16). 


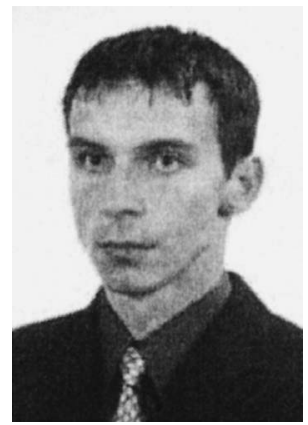

Pawet Olejnik was born in Zduńska Wola, Poland on August 29, 1975. He was graduated from the Faculty of Technical Physics Computer Science and Applied Mathematics of the Technical University of Łódź. He received the Ph.D. Eng. degree in a field of Mechanics from the same university in 2002. Since 2000, he has worked in the Department of Automatics and Biomechanics and, since 2002, he has been Coordinator of the Erasmus-Socrates program at the Faculty of Mechanical Engineering. He is coauthor of 15 publications in scientific journals and conference proceedings. His research is oriented on the natural sciences like physics, mathematics, and mechanics, putting special emphasis on investigations of friction phenomena. 